\title{
Density functional theory for colloidal mixtures of hard platelets, rods, and spheres
}

\author{
Ansgar Esztermann ${ }^{1}$, Hendrik Reich ${ }^{1}$, and Matthias Schmidt ${ }^{1,2}$ \\ ${ }^{1}$ Institut für Theoretische Physik II, \\ Heinrich-Heine-Universität Düsseldorf, \\ Universitätsstraße 1, D-40225 Düsseldorf, Germany and \\ ${ }^{2}$ H. H. Wills Physics Laboratory, University of Bristol, \\ Royal Fort, Tyndall Avenue, Bristol BS8 1TL, UK
}

(Dated: 18 November 2005)

\begin{abstract}
A geometry-based density functional theory is presented for mixtures of hard spheres, hard needles and hard platelets; both the needles and the platelets are taken to be of vanishing thickness. Geometrical weight functions that are characteristic for each species are given and it is shown how convolutions of pairs of weight functions recover each Mayer bond of the ternary mixture and hence ensure the correct second virial expansion of the excess free energy functional. The case of sphere-platelet overlap relies on the same approximation as does Rosenfeld's functional for strictly two-dimensional hard disks. We explicitly control contributions to the excess free energy that are of third order in density. Analytic expressions relevant for the application of the theory to states with planar translational and cylindrical rotational symmetry, e.g. to describe behavior at planar smooth walls, are given. For binary sphere-platelet mixtures, in the appropriate limit of small platelet densities, the theory differs from that used in a recent treatment [L. Harnau and S. Dietrich, Phys. Rev. E 71, 011504 (2004)]. As a test case of our approach we consider the isotropic-nematic bulk transition of pure hard platelets, which we find to be weakly first order, with values for the coexistence densities and the nematic order parameter that compare well with simulation results.
\end{abstract}

PACS numbers: 61.20.Gy, 82.70.Dd, 61.30.Cz 


\section{INTRODUCTION}

Dispersions of non-spherical colloidal particles are model systems to study a broad range of phenomena in condensed matter ranging from fluid phase separation to the emergence of liquid crystalline ordering. Examples of such systems are clay suspensions [1, 2], dispersed gibbsite platelets [3], mixtures of silica spheres and silica-coated boehmite rods [4], wax disks [5], or nonaqueous suspensions of laponite and montmorillonite [6]. While already pure systems often possess complex liquid crystal phase behavior, binary mixtures additionally may demix into bulk phases with different chemical compositions, e.g. like in dispersions of disks and spheres [5]. Such mixtures are often viewed as composed of a primary species (here the spheres) that interact with an effective depletion potential that is generated by the second component referred to as the depletion agent (here the disks). The depletion interaction is primarily attractive with a range of attraction similar to the size of the depletant agent and with a strength that is ruled by the concentration of the depletion agent. Under appropriate conditions the depletion interaction may be sufficiently strong in order to drive a phase transition that is anaologous to the gas-liquid phase separation in simple substances, and that manifests fluid-fluid demixing when regarding the full mixture.

Recent theoretical work has been devoted to fluids of platelike particles near a hard wall 7], an interaction site model for lamellar colloids [8], wetting and capillary nematization of binary hard-platelet and hard-rod fluids [9], the lamellar Zwanzig model [10, 11], and colloidal hard-rod fluids near geometrically structured substrates [12]. The phase diagram of mixtures of hard colloidal spheres and discs was obtained within a free-volume scaled particle approach [13]. The depletion potential between two spheres immersed in a sea of platelets was studied in detail [14, 15], and found to compare well with predictions from the Derjaguin approximation [14]. Sedimentation was found to influence liquid crystal phase transitions of colloidal platelets [16], as well as multi-phase equilibria in mixtures of platelets and ideal polymer [17].

The fundamental-measure theory (FMT) is an (approximate) density-functional theory (DFT) [18, 19], originally proposed by Rosenfeld for additive hard sphere mixtures [20]. An early extension to convex non-spherical particles has been given in Refs. 21, 22]. When applied to homogeneous and isotropic fluid states, this theory yields the correct second virial coefficient of the equation of state, but fails to recover the exact density functional up to 
second order in density. To remedy the latter problem, an interpolation between the hard sphere Rosenfeld functional and the Onsager functional for elongated rods was proposed 23] and applied to the bulk isotropic-nematic transition. An FMT that originated from the treatment of parellel hard cubes [24, 25, 26] was also used very successfully for the Zwanzig model, see e.g. [27, 28]. See also Refs. [29, 30, 31] for the treatment of various hard body system mixtures. Recently attention was paid to binary mixtures of hard rods and polymers 32, 33]. Also notable is the treatment of hard body fluids based on two-point measures [34, 35, 36].

The Bolhuis-Frenkel model of hard spheres and vanishingly thin hard needles [37] can be considered as the simplest model hard core mixture of spheres and rods. Previous work was concerned with the formulation of a DFT for this model [38], and an extension to include rod-rod interaction on the Onsager (second virial) level [39, 40]. Predictions for the orientation ordering of the rods at a free interface between (isotropic) sphere-rich and sphere-poor phases [39] were successfully confirmed by simulations [41]. Adding a third component to this system, the fluid demixing phase behavior of ternary mixtures of spheres, rods, and model polymers was found to be rich [42]. Quenching one of the components led to investigations of hard spheres immersed in random rod networks [43], and of the isotropic-nematic transition of rods in matrices of immobilized spheres [44]. The present paper is concerned with the introduction of hard platelets to the FMT framework.

Central to FMT is the so-called deconvolution of the Mayer bond $f_{i j}$ between particles of species $i$ and $j$ into "weight functions" $w_{i}^{\nu}$ that are characteristic of the shape of particles of each species $i$; the label $\nu$ indicates the type of weight function. Recall that the Mayer bond is related to the pair interaction potential $V_{i j}$ between species $i$ and $j$ via $f_{i j}=\exp \left(-\beta V_{i j}\right)-$ 1 , where $\beta=1 /\left(k_{B} T\right), k_{B}$ is the Boltzmann constant and $T$ is temperature. For hard bodies $f_{i j}=-1$ if the two particles overlap and $f_{i j}=0$ otherwise. In the FMT framework a convolution integral is used to express $f_{i j}$ as a sum of terms each of which is bilinear in the weight functions, i.e. possesses the structure $w_{i}^{\nu} * w_{j}^{\tau}$, where $i, j$ label the species, $\nu, \tau$ label the type of weight function, and the asterisk indicates the (three-dimensional) convolution. The weight functions are characteristic of the geometry of single particles, i.e. vanish (already) beyond the physical extent of one particle. Note that this is different from the behavior of $f_{i j}$, which extends to larger separations and vanishes provided two particles do not overlap. However the convolution of pairs of (shorter ranged) weight functions restores 
the correct (longer ranged) non-locality of the Mayer bond. This concept was originally introduced for hard sphere mixtures [20]. An extension to arbitrarily shaped convex bodies was proposed in Refs. [21, 22], which yields the correct second virial coefficients, but gives only an approximation for the Mayer bonds. This is insufficient to describe e.g. nematic ordering (see e.g. Ref. 23] for a discussion). However, obtaining exact deconvolutions is possible in principle, as the cases of the Mayer bond between a sphere and a vanishingly thin needle [38] and between a pair of thin rods [39] demonstrate.

To give an overview of the general structure of FMT, the weight functions $w_{i}^{\tau}$ are used to to build so-called weighted densities via convolution with the bare one-body density distribution (of each species in the case of a mixture). The weighted densities are then input as arguments to an (analytically given) excess free energy density, $\Phi$. Integrating $\Phi$ over space (and director space in case of anisotropic particles) yields the excess free energy of the system, which is the only unknown contribution to the grand potential functional being the central quantity in (classical) DFT. For any external potential (like describing gravity or a wall) minimizing the grand potential with respect to the one-body density distribution(s) gives both the value of the grand potential and the structural information contained in the density profile(s).

Here we show how to treat platelet-shaped particles and mixtures with both spheres and needles within the same framework. The relevant weight functions are given and it is shown explicitly how all Mayer bonds for the ternary mixture are obtained through convolutions. For two-dimensional hard disks the Rosenfeld functional only yields an approximation for the exact Mayer bond. Despite this deficieny the two-dimensional Rosenfeld functional is considered to be a useful tool to study inhomogeneous situations, see e.g. Ref. [45] for an investigation of laser-induced freezing and melting of confined colloidal particles. The treatment of the sphere-platelet case inevitably leads to the same defect. We do achieve, however, exact deconvolutions of the Mayer bonds for platelet-platelet and platelet-needle interactions.

Furthermore we treat explicitly contributions to the excess free energy functional that are of third order in densities. While the treatment of second order terms was a crucial input for the original construction of FMT for hard spheres [20], this route has been only recently pursued in detail [46], and the authors conclude that they come "close to the edge of FMT" in attempting to incorporate properties of the exact expansion of the free energy 
functional in density on the third virial level into the approximate framework of FMT. In particular these delicate cases appear when particle touch, i.e. configurations that are close to the edge of the particles.

Hence we approximate the exact third virial level (the triangle in the diagrammatic Mayer $f_{i j}$-expansion) by terms that are non-vanishing only for cases with common triple intersection of the three particles involved. Global prefactors are used to compensate for the "lost cases" [46, 47] in order to yield reasonable third virial coefficients.

As a test case for the accuracy of the theory we consider the pure system of hard platelets. This is known to undergo a weak first order isotropic-nematic phase transition [48]. In contrast to the application to thin rods, the Onsager (second virial) functional for platelets yields only a qualitatively correct account of the phase transition: The transition density, density jump, and value of the nematic order parameter in the coexisting nematic are significantly overestimated. Our theory has the same (exact) second order contribution to the free energy, but also sports a further third-order term. Higher than third-order terms are absent due to the scaled-particle roots [54] of the approach; the vanishing thickness and hence vanishing volume of the platelets effectively truncates the series. Our results show that FMT considerably improves on the Onsager treatment, and yields a quantitatively correct picture, albeit with slightly too small transitition densities.

Harnau and Dietrich recently considered bulk and wetting phenomena in a binary mixture of colloidal hard spheres and hard platelets [49], an interesting limiting case of the present ternary mixture. We postpone a detailed discussion of the relationship of the current work to Ref. [4] to Sec. [X]

The paper is organized as follows. In Sec. II we define the model of a ternary hard body mixture of spheres, needles, and platelets. In Sec. III the Mayer bonds are represented as convolutions of (weight) functions characteristic for single particles. In Sec. [V] we proceed to control the third virial level. Readers who are interested in the structure of the resulting theory rather than its derivation might wish to skip Secs. III and IV and directly go to Sec. $\nabla$ where the weighted densities are defined as convolutions of the weight functions with the bare density profiles. Sec. VI presents the excess free energy functional. Explicit expressions for the relevant quantities in planar and uniaxial geometry are given in Sec. VII We apply the theory to the isotropic-nematic transition of hard platelets in Sec. VIII, and conclude in Sec. IX 


\section{MODEL}

We consider a mixture of hard spheres (species $S$ ) with diameter $\sigma=2 R$, where $R$ is the radius, hard platelets (species $D$ ) of diameter $2 R_{D}$ and vanishing thickness, and hard needlelike rods (species $N$ ) of length $L$ and vanishing thickness. The pair interaction potential $V_{i j}$ between any two particles of species $i, j=S, D, N$ is infinite if their geometrical shapes overlap and zero otherwise. The one-body density distribution of species $i=S, D, N$ is denoted by $\rho_{S}(\mathbf{r}), \rho_{D}(\mathbf{r}, \Omega)$, and $\rho_{N}(\mathbf{r}, \Omega)$, respectively, where $\mathbf{r}$ is the position of the particle center, and $\Omega$ is a unit vector pointing along (normal to) the shape of the needle (platelet) describing the particle orientation in space.

\section{DECONVOLUTION OF THE MAYER BONDS}

\section{A. The sphere-sphere and sphere-needle Mayer bonds}

For completeness we first summarize results from the literature for hard spheres and their mixtures with needles. Rosenfeld's hard sphere weight functions [20] are

$$
\begin{aligned}
w_{3}^{S}(\mathbf{r}) & =\Theta(R-|\mathbf{r}|), \\
w_{2}^{S}(\mathbf{r}) & =\delta(R-|\mathbf{r}|), \\
\mathbf{w}_{v 2}^{S}(\mathbf{r}) & =w_{2}^{S}(\mathbf{r}) \frac{\mathbf{r}}{|\mathbf{r}|},
\end{aligned}
$$

where $\Theta(\cdot)$ is the unit step (Heaviside) function and $\delta(\cdot)$ is the Dirac distribution. Here and in the following the $w_{\tau}^{i}$ are quantities with the dimension of (length) ${ }^{\tau-3}$; the subscript $v$ indicates a vectorial quantity. Further, linearly dependent, weight functions are $w_{1}^{S}(\mathbf{r})=$ $w_{2}^{S}(\mathbf{r}) /(4 \pi R), w_{0}^{S}(\mathbf{r})=w_{2}^{S}(\mathbf{r}) /\left(4 \pi R^{2}\right)$, and $\mathbf{w}_{v 1}^{S}(\mathbf{r})=\mathbf{w}_{v 2}^{S}(\mathbf{r}) /(4 \pi R)$. For pure hard spheres the Mayer bond is obtained through

$$
\begin{gathered}
-f_{S S}(\mathbf{r}) / 2=w_{3}^{S}(\mathbf{r}) * w_{0}^{S}(\mathbf{r})+w_{2}^{S}(\mathbf{r}) * w_{1}^{S}(\mathbf{r}) \\
-\mathbf{w}_{v 2}^{S}(\mathbf{r}) * \mathbf{w}_{v 1}^{S}(\mathbf{r})
\end{gathered}
$$

where the (three-dimensional) convolution is defined as

$$
h(\mathbf{r}) * g(\mathbf{r})=\int \mathrm{d}^{3} x h(\mathbf{x}) g(\mathbf{x}-\mathbf{r})
$$


and also implies a scalar product between vectors, as appears in the last term on the right hand side of Eq. (4).

A pair of vanishingly thin needles does not experience excluded volume interactions, but needles do interact with hard spheres. For such a binary mixture the needle weight functions used in Ref. [38] were obtained from the prescription of Refs. [21, 22], and are given by

$$
\begin{aligned}
& w_{0}^{N}(\mathbf{r}, \boldsymbol{\Omega})=\frac{1}{2}\left[\delta\left(\mathbf{r}-\frac{L}{2} \boldsymbol{\Omega}\right)+\delta\left(\mathbf{r}+\frac{L}{2} \boldsymbol{\Omega}\right)\right], \\
& w_{1}^{N}(\mathbf{r}, \boldsymbol{\Omega})=\frac{1}{4} \int_{-L / 2}^{L / 2} \mathrm{~d} l \delta(\mathbf{r}-l \boldsymbol{\Omega}) .
\end{aligned}
$$

Introducing a "mixed" weight function that is non-vanishing on the surface of a sphere, but carries a dependence on orientation,

$$
w_{2}^{S N}(\mathbf{r}, \boldsymbol{\Omega})=2\left|\mathbf{w}_{v 2}^{S}(\mathbf{r}) \cdot \boldsymbol{\Omega}\right|,
$$

allows one to obtain the Mayer bond between sphere and needle through convolutions,

$$
-f_{S N}(\mathbf{r}, \boldsymbol{\Omega})=w_{3}^{S}(\mathbf{r}) * w_{0}^{N}(\mathbf{r}, \boldsymbol{\Omega})+w_{2}^{S N}(\mathbf{r}, \boldsymbol{\Omega}) * w_{1}^{N}(\mathbf{r}, \boldsymbol{\Omega}),
$$

see Ref. [39] for the explicit calculation. For the case of residual rod-rod interactions in the Onsager limit, $f_{N N}$ can also be deconvolved into weight functions, see Ref. [39, 40] for the details. In the following, however, we will restrict ourselves to the case of vanishingly thin needles.

\section{B. Strictly two-dimensional hard disks}

As a prerequisite for our subsequent treatment of platelets in three dimensions, we give an overview of Rosenfeld's functional for the model of strictly two-dimensional disks. This constitues a prerequisite for considering the overlap between a hard sphere and a hard platelet in three dimensions (Sec. IIIE below). Here we deal with a multicomponent twodimensional mixture of disks with radii $R_{i}$ of species $i$, and characterized by the pair potential $V_{i j}(r)=\infty$ if $r<R_{i}+R_{j}$ and zero otherwise, where $r$ is the center-center distance between the disks of species $i$ and $j$. (When regarded from the viewpoint of the three-dimensional model, all disks possess the same orienation $\Omega$, perpendicular to the two-dimensional plane of position coordinates; we will not delve into this delicate dimensional crossover in the 
following). Rosenfeld's weight functions for this model are

$$
\begin{aligned}
w_{2}^{(i)}(\mathbf{r}) & =\Theta\left(R_{i}-|\mathbf{r}|\right), \\
w_{1}^{(i)}(\mathbf{r}) & =\delta\left(R_{i}-|\mathbf{r}|\right), \\
\mathbf{w}_{v 1}^{(i)}(\mathbf{r}) & =w_{2}^{(i)}(\mathbf{r}) \frac{\mathbf{r}}{|\mathbf{r}|},
\end{aligned}
$$

and there is an additional linearly dependent weight function, $w_{0}^{(i)}(\mathbf{r})=w_{2}^{(i)}(\mathbf{r}) /\left(2 \pi R_{i}\right)$. The exact Mayer bond $f_{i j}$ between species $i$ and $j$ is then approximated through $f_{i j} \approx f_{i j}^{*}$, with

$$
\begin{aligned}
-f_{i j}^{*}(r)= & w_{0}^{(i)}(\mathbf{r}) * w_{2}^{(j)}(\mathbf{r})+w_{2}^{(i)}(\mathbf{r}) * w_{0}^{(j)}(\mathbf{r}) \\
& +\frac{1}{2 \pi}\left(w_{1}^{(i)}(\mathbf{r}) * w_{1}^{(j)}(\mathbf{r})-\mathbf{w}_{v 1}^{(i)}(\mathbf{r}) * \mathbf{w}_{v 1}^{(j)}(\mathbf{r})\right),
\end{aligned}
$$

where here (and only here) * denotes the two-dimensional convolution,

$$
h(\mathbf{r}) * g(\mathbf{r})=\int \mathrm{d}^{2} x h(\mathbf{x}) g(\mathbf{x}-\mathbf{r})
$$

and again the convolution implies a scalar product between vectors.

As our subsequent treatment of the three-dimensional sphere-platelet overlap relies heavily on Eq. (12), we calculate the explicit functional dependence of $f_{i j}^{*}(r)$ on $r$ and discuss some of its properties. We choose two-dimensional Cartesian coordinates $\mathbf{r}$ and $\mathbf{x}$, such that $\mathbf{r}=(0, r)$ and $\mathbf{x}=\left(x^{\prime} \sin \varphi^{\prime}, x^{\prime} \cos \varphi^{\prime}\right)$, which allows us to write the first term on the r.h.s. of Eq. (12) as

$$
\begin{aligned}
\int \mathrm{d}^{2} x w_{0}^{(i)}(\mathbf{x}) & w_{2}^{(j)}(\mathbf{x}-\mathbf{r}) \\
& =\int_{0}^{2 \pi} \mathrm{d} \varphi^{\prime} \int_{0}^{\infty} x^{\prime} \mathrm{d} x^{\prime} \frac{1}{2 \pi R_{i}} \delta\left(x^{\prime}-R_{i}\right) \Theta\left(R_{j}-\sqrt{x^{\prime 2}+r^{2}-2 r x^{\prime} \cos \varphi^{\prime}}\right) \\
& =\frac{1}{\pi} \arccos \left(\frac{r^{2}+R_{i}^{2}-R_{j}^{2}}{2 r R_{i}}\right) \Theta\left(2 r R_{i}-\left|r^{2}+R_{i}^{2}-R_{j}^{2}\right|\right),
\end{aligned}
$$

which is, by the cosine theorem, equal (up to a factor of $2 \pi$ ) to the length of the arc which the rim of the disc $i$ traces across the interior of disc $j$ (see Fig. 1). By symmetry, the second term on the r.h.s. of Eq. (12) gives Eq. (14) with $R_{i}$ and $R_{j}$ interchanged. To calculate the 
remaining third term on the r.h.s. of Eq. (12), we use the same setup as above and obtain

$$
\begin{aligned}
\frac{1}{2 \pi} \int \mathrm{d}^{2} x\left(w_{1}^{(i)}(\mathbf{x}) w_{1}^{(j)}\right. & \left.(\mathbf{x}-\mathbf{r})-\mathbf{w}_{v 1}^{(i)}(\mathbf{x}) \cdot \mathbf{w}_{v 1}^{(j)}(\mathbf{x}-\mathbf{r})\right) \\
= & \frac{1}{2 \pi} \int_{0}^{2 \pi} \mathrm{d} \varphi^{\prime} \int_{0}^{\infty} \mathrm{d} x^{\prime} x^{\prime} \delta\left(R_{i}-x^{\prime}\right) \delta\left(R_{j}-\sqrt{r^{2}+x^{\prime 2}-2 r x^{\prime} \cos \varphi^{\prime}}\right) \\
& \times\left(1-\frac{1}{R_{i} R_{j}}\left(\begin{array}{c}
x^{\prime} \sin \varphi^{\prime} \\
x^{\prime} \cos \varphi^{\prime}
\end{array}\right) \cdot\left(\begin{array}{c}
x^{\prime} \sin \varphi^{\prime} \\
x^{\prime} \cos \varphi^{\prime}-r
\end{array}\right)\right) \\
= & \frac{1}{\pi} \Theta\left(R_{i}-\left|R_{j}-r\right|\right) \frac{r^{2}-\left(R_{i}-R_{j}\right)^{2}}{\sqrt{4 r^{2} R_{i}^{2}-\left(r^{2}+R_{i}^{2}-R_{j}^{2}\right)^{2}}}
\end{aligned}
$$

In the special case that both platelets have the same radius, $R_{i}=R_{j}$, as relevant for describing the pure hard disk system, Eq. (16) further simplifies to

$$
\frac{1}{2 \pi} \int \mathrm{d}^{2} x\left(w_{1}^{(i)}(\mathbf{x}) w_{1}^{(i)}(\mathbf{x}-\mathbf{r})-\mathbf{w}_{v 1}^{(i)}(\mathbf{x}) \cdot \mathbf{w}_{v 1}^{(i)}(\mathbf{x}-\mathbf{r})\right)=\Theta\left(2 R_{i}-r\right) \frac{r}{\pi \sqrt{4 R_{i}^{2}-r^{2}}}
$$

We plot $f_{i j}^{*}(r)$ in Fig. 2 for three typical values of the size ratio $R_{i} / R_{j}=0.44,0.71,1$. For small separations $r$, such that the (small) disk $i$ lies completely inside the (big) disk $j$, the result is exact, $f_{i j}^{*}(r)=-1$ for $r<R_{j}-R_{i}$. As $r$ increases, $-f_{i j}^{*}$ first decreases and then increases, exhibiting a divergence at contact, $r=R_{i}+R_{j}$. Beyond this threshold, for $r>R_{i}+R_{j}, f_{i j}^{*}$ is (correctly) indentically zero. Note that the deviations around the exact value inside the core, $f_{i j}=-1$, balance such that the second virial coefficient is exact, $\int d^{2} r f_{i j}(r)=\int d^{2} r f_{i j}^{*}(r)=-\pi\left(R_{i}+R_{j}\right)^{2}$.

\section{Platelet-platelet Mayer bond in three dimensions}

Although the particle shapes appear to be equivalent, overlapping pair configurations are very different for freely rotating platelets in three dimensions as compared to the strictly twodimensional case. Note that in three dimensions we only need to consider cases of differing orientations of both platelets, $\Omega \neq \Omega^{\prime}$, as configurations with strictly equal orientations, $\Omega=\Omega^{\prime}$, carry vanishing statistical weight. The intersection volume differs markedly from the two-dimensional case (area of intersection), requiring very different weight functions to express the Mayer bond. This task can be accomplished exactly, in contrast to the twodimensional case above. The direct application of Rosenfeld's recipe [21, 22] to platelets 


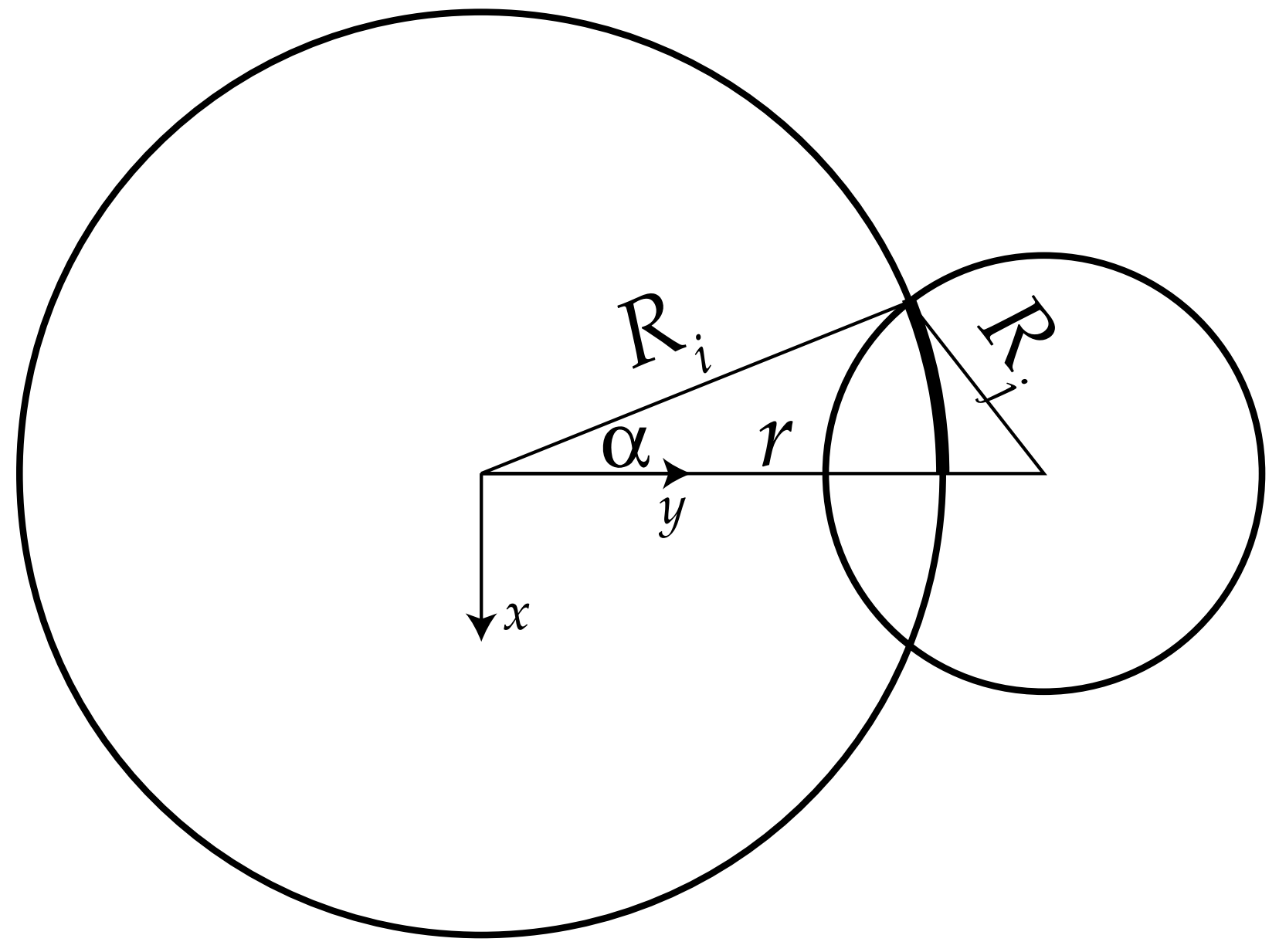

FIG. 1: Overlapping pair of strictly two-dimensional hard disks with radii $R_{i}$ and $R_{j}$ and centercenter distance $r$. Shown are the $x$ and $y$ axis of the coordinate system used, the angle $\alpha$ between the y-axis and $R_{i}$, as well as half of the part (bold) of the circumference of disk $i$ that lies inside disk $j$.

yields the scalar weight functions

$$
\begin{aligned}
& w_{2}^{D}(\mathbf{r}, \boldsymbol{\Omega})=2 \Theta\left(R_{D}-|\mathbf{r}|\right) \delta(\mathbf{r} \cdot \boldsymbol{\Omega}) \\
& w_{1}^{D}(\mathbf{r}, \boldsymbol{\Omega})=\delta\left(R_{D}-|\mathbf{r}|\right) \delta(\mathbf{r} \cdot \boldsymbol{\Omega}) / 8 \\
& w_{0}^{D}(\mathbf{r}, \boldsymbol{\Omega})=\frac{1}{2 \pi R_{D}} \delta\left(R_{D}-|\mathbf{r}|\right) \delta(\mathbf{r} \cdot \boldsymbol{\Omega}),
\end{aligned}
$$




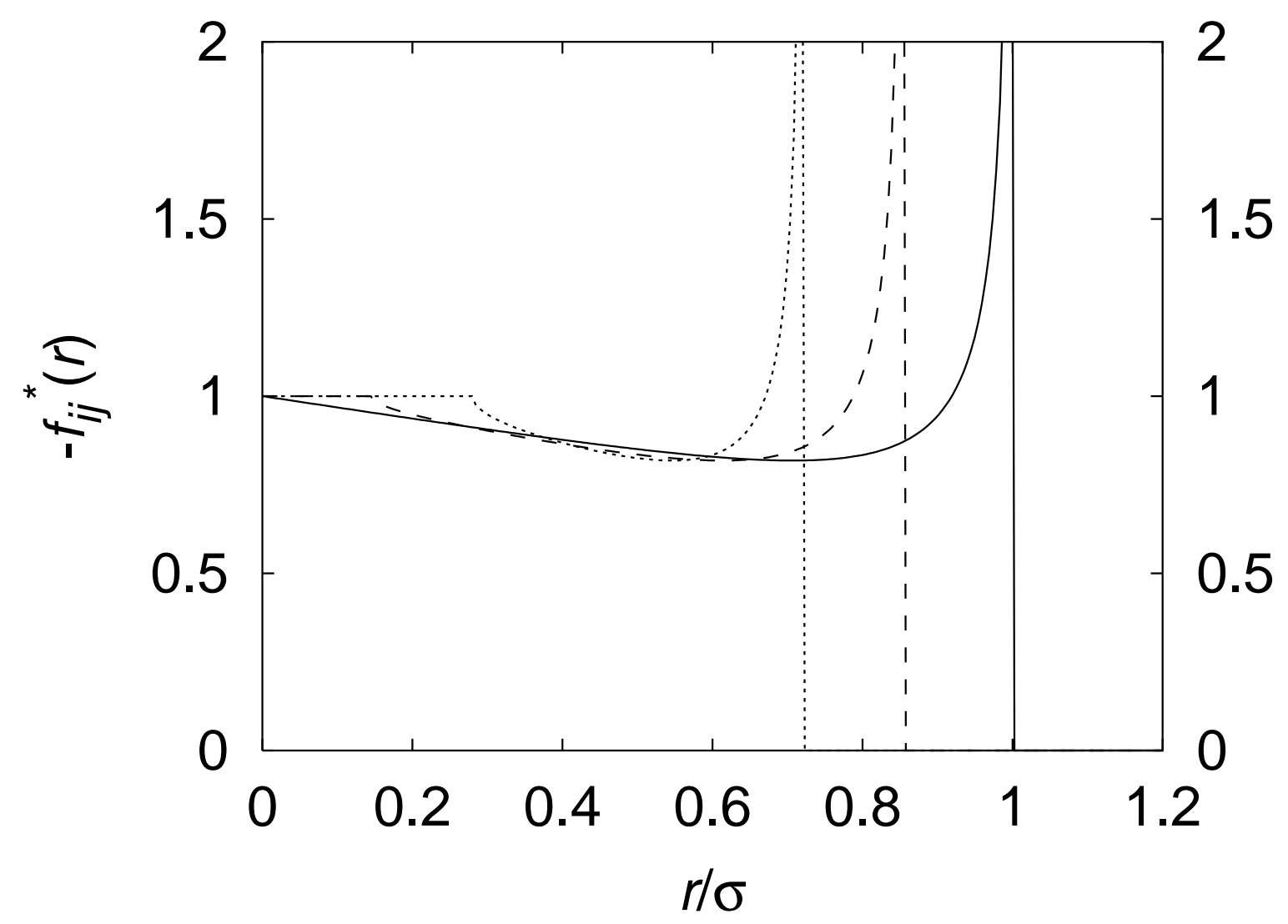

FIG. 2: Rosenfeld's approximation for the (negative) Mayer bond, $-f_{i j}^{*}(r)$, of two-dimensional hard disks of radii $R_{i}$ and $R_{j}$, for size ratios $R_{i} / R_{j}=0.44,0.71,1$ (from left to right) as a function of the (scaled) center-to-center distance $r / \sigma$, where $\sigma=2 R_{j}$. In Sec. IIIE the same result is obtained for the Mayer bond in three dimensions between a sphere of radius $R$ and a platelet of radius $R_{D}$ as a function of the (scaled) distance $r / \sigma$ between the center of the platelet and the axis of the sphere (parallel to the orientation $\Omega$ of the platelet), where $\sigma=2 R_{D}$. The (scaled) distance between the center of the sphere and the plane of the platelet is $0,0.7,0.9$ (right to left), and $R=R_{D}$. The result for the Mayer bond obtained is exact in cases (not shown) where the platelet i) fully cuts through the sphere or ii) lies completely inside the sphere (provided $R_{D}<R$ ).

corresponding to the surface, $\xi_{2}^{D}=2 \pi R_{D}^{2}$, integral mean curvature, $\xi_{1}^{D}=\pi R_{D} / 4$, and Euler characteristic, $\xi_{0}^{D}=1$, of the platelets, respectively. The weight function that describes the particle volume vanishes, $w_{3}^{S}(\mathbf{r}, \boldsymbol{\Omega})=0$, due to the vanishing thickness, and hence vanishing volume of the platelets, $\xi_{3}^{D}=0$.

In contrast to Rosenfeld's treatment [21, 22], we here aim at an exact deconvolution of 
$f_{D D}\left(\mathbf{r}, \Omega^{\prime}, \Omega\right)$, where $\mathbf{r}$ is the center-center distance vector between both particles and $\boldsymbol{\Omega}$ and $\Omega^{\prime}$ are their orientations. We introduce

$$
w_{1}^{D D}\left(\mathbf{r}, \boldsymbol{\Omega} ; \boldsymbol{\Omega}^{\prime}\right)=\frac{2}{R_{D}}\left|\boldsymbol{\Omega} \cdot\left(\boldsymbol{\Omega}^{\prime} \times \mathbf{r}\right)\right| w_{1}^{D}(\mathbf{r}, \boldsymbol{\Omega}),
$$

where $w_{1}^{D}(\mathbf{r}, \boldsymbol{\Omega})$, given through Eq. (19), describes the platelet rim, and $\boldsymbol{\Omega}^{\prime}$ is the orientation of the second platelet. Keeping Rosenfeld's surface weight function, $w_{2}^{D}(\mathbf{r}, \boldsymbol{\Omega})$ as given in Eq. (18), we recover the Mayer bond between platelets via convolution,

$$
\begin{aligned}
-f_{D D}\left(\mathbf{r}, \boldsymbol{\Omega} ; \boldsymbol{\Omega}^{\prime}\right)= & w_{1}^{D D}\left(\mathbf{r}, \boldsymbol{\Omega} ; \boldsymbol{\Omega}^{\prime}\right) * w_{2}^{D}\left(\mathbf{r}, \boldsymbol{\Omega}^{\prime}\right) \\
& +w_{2}^{D}(\mathbf{r}, \boldsymbol{\Omega}) * w_{1}^{D D}\left(\mathbf{r}, \boldsymbol{\Omega}^{\prime} ; \boldsymbol{\Omega}\right) .
\end{aligned}
$$

As the r.h.s. of Eq. (22) consists of two symmetric terms, it is sufficient to consider the first one; the second one gives an equivalent contribution to $f_{D D}$. Without loss of generality we place the particles such that one platelet is located at the origin with its orientation vector pointing up (into the positive $z$-direction), and place the center of the other platelet in the $y-z$ plane; see Fig. [ 3 for an illustration. The chosen coordinates are: $\mathbf{r}=(0, r, z)$, $\boldsymbol{\Omega}=(0,0,1), \boldsymbol{\Omega}^{\prime}=\left(\sqrt{1-\bar{z}^{2}} \sin \bar{\varphi}, \sqrt{1-\bar{z}^{2}} \cos \bar{\varphi}, \bar{z}\right)$, and the integration variable is $\mathbf{x}=$ $\left(r^{\prime} \sin \varphi^{\prime}, r^{\prime} \cos \varphi^{\prime}, z^{\prime}\right)$. Using (19) and (21) we insert the explicit form $w_{1}^{D D}\left(\mathbf{r}, \boldsymbol{\Omega} ; \boldsymbol{\Omega}^{\prime}\right)=$ 


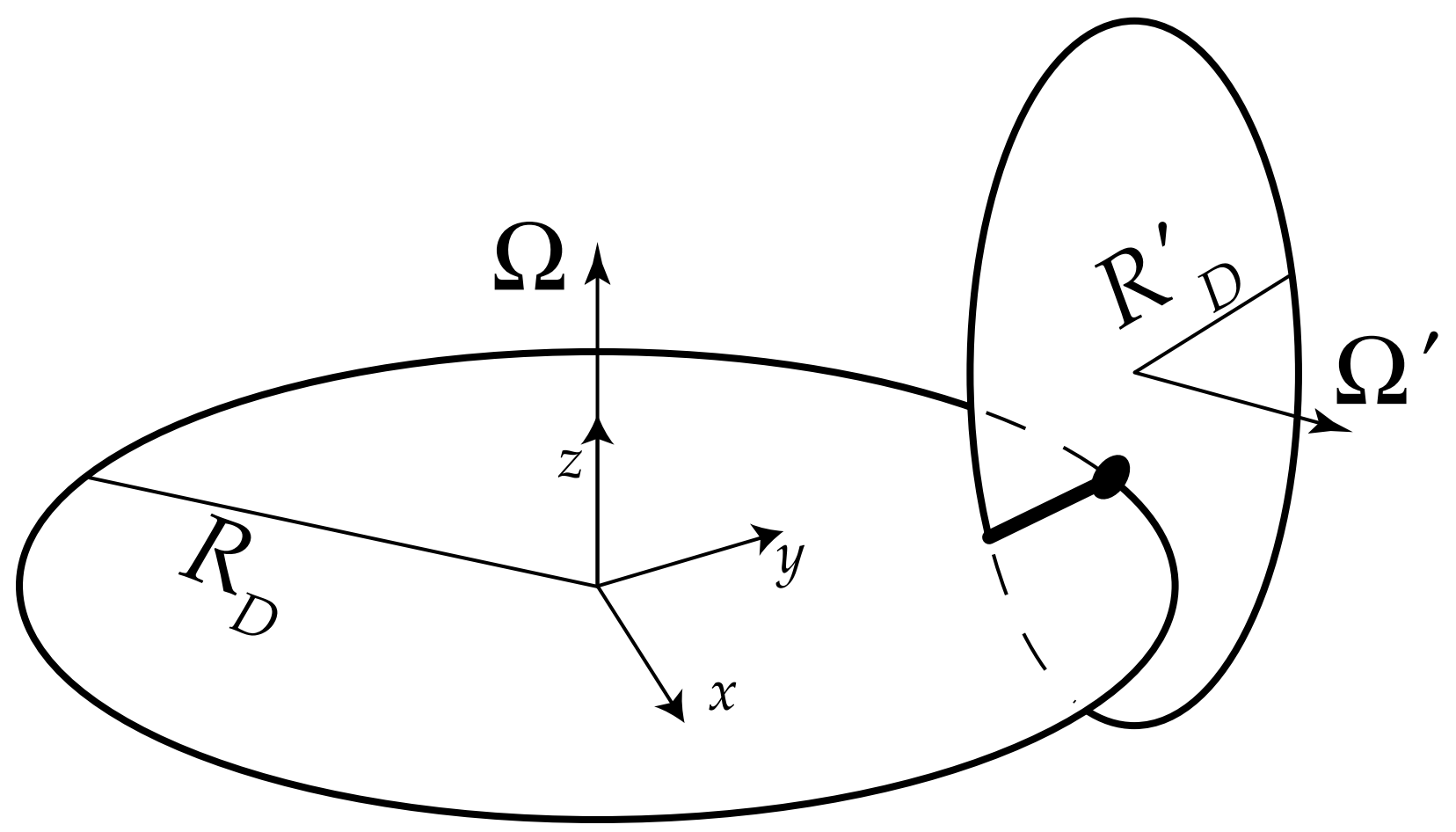

FIG. 3: Setup for the calculation of the platelet-platelet Mayer bond. Platelet $D$ is located in the $x-y$ plane with its center at the origin. Platelet $D^{\prime}$ has its center in the $y-z$ plane and an arbitrary orientation. The calculation checks for the intersection of the rim of $D$ and the interior of $D^{\prime}$ (bold dot). The one-dimensional intersection volume of the two platelets is shown as a bold line. 
$\left|\mathbf{\Omega}^{\prime} \cdot(\mathbf{r} \times \mathbf{\Omega})\right| \delta\left(R_{D}-|\mathbf{r}|\right) \delta(\mathbf{r} \cdot \boldsymbol{\Omega}) /\left(8 R_{D}\right)$ into the first term on the r.h.s. of (22) to obtain

$$
\begin{aligned}
& \int \mathrm{d}^{3} x w_{1}^{D D}\left(\mathbf{x}, \mathbf{\Omega} ; \mathbf{\Omega}^{\prime}\right) w_{2}^{D}\left(\mathbf{x}-\mathbf{r}, \mathbf{\Omega}^{\prime}\right) \\
& =\frac{1}{2 R_{D}} \int_{0}^{2 \pi} \mathrm{d} \varphi^{\prime} \int_{-\infty}^{\infty} \mathrm{d} z^{\prime} \int_{0}^{\infty} \mathrm{d} r^{\prime} r^{\prime}\left|\left(\begin{array}{l}
0 \\
0 \\
1
\end{array}\right) \cdot\left[\left(\begin{array}{c}
\sqrt{1-\bar{z}^{2}} \sin \bar{\varphi} \\
\sqrt{1-\bar{z}^{2}} \cos \bar{\varphi} \\
\bar{z}
\end{array}\right) \times\left(\begin{array}{c}
r^{\prime} \sin \varphi^{\prime} \\
r^{\prime} \cos \varphi^{\prime} \\
z^{\prime}
\end{array}\right)\right]\right| \\
& \times \delta\left(R_{D}-\sqrt{r^{\prime 2}+z^{\prime 2}}\right) \delta\left(z^{\prime}\right) \Theta\left(R_{D}-\left|\left(r^{\prime} \sin \varphi^{\prime}, r^{\prime} \cos \varphi^{\prime}-r, z^{\prime}-z\right)\right|\right) \\
& \times \delta\left(\left(\begin{array}{c}
r^{\prime} \sin \varphi^{\prime} \\
r^{\prime} \cos \varphi^{\prime}-r \\
z^{\prime}-z
\end{array}\right) \cdot\left(\begin{array}{c}
\sqrt{1-\bar{z}^{2}} \sin \bar{\varphi} \\
\sqrt{1-\bar{z}^{2}} \cos \bar{\varphi} \\
\bar{z}
\end{array}\right)\right) \\
& =\frac{R_{D}}{2} \int_{0}^{2 \pi} \mathrm{d} \varphi^{\prime}\left|\left(\begin{array}{c}
\sin \varphi^{\prime} \\
\cos \varphi^{\prime} \\
0
\end{array}\right) \cdot\left(\begin{array}{c}
-\sqrt{1-\bar{z}^{2}} \cos \bar{\varphi} \\
\sqrt{1-\bar{z}^{2}} \sin \bar{\varphi} \\
0
\end{array}\right)\right| \\
& \times \Theta\left(R_{D}-\left|\left(R_{D} \sin \varphi^{\prime}, R_{D} \cos \varphi^{\prime}-r,-z\right)\right|\right) \\
& \times \delta\left(\left(\begin{array}{c}
R_{D} \sin \varphi^{\prime} \\
R_{D} \cos \varphi^{\prime}-r \\
-z
\end{array}\right) \cdot\left(\begin{array}{c}
\sqrt{1-\bar{z}^{2}} \sin \bar{\varphi} \\
\sqrt{1-\bar{z}^{2}} \cos \bar{\varphi}
\end{array}\right)\right) \\
& =\frac{R_{D}}{2} \int_{0}^{2 \pi} \mathrm{d} \varphi^{\prime}\left|\sqrt{1-\bar{z}^{2}} \sin \left(\bar{\varphi}-\varphi^{\prime}\right)\right| \Theta\left(2 r R_{D} \cos \varphi^{\prime}-r^{2}-z^{2}\right) \\
& \times \delta\left(r \sqrt{1-\bar{z}^{2}} \cos \bar{\varphi}-R_{D} \sqrt{1-\bar{z}^{2}} \cos \left(\varphi^{\prime}-\bar{\varphi}\right)+z \bar{z}\right) \\
& =\frac{1}{2} \sum_{ \pm} \Theta\left(2 r R_{D} \cos \left(\bar{\varphi} \pm \arccos \frac{r \sqrt{1-\bar{z}^{2}} \cos \bar{\varphi}+z \bar{z}}{R_{D} \sqrt{1-\bar{z}^{2}}}\right)-r^{2}-z^{2}\right) \\
& \times \Theta\left(R_{D} \sqrt{1-\bar{z}^{2}}-\left|r \sqrt{1-\bar{z}^{2}} \cos \bar{\varphi}+z \bar{z}\right|\right),
\end{aligned}
$$

where the integrations over $z^{\prime}$ and $r^{\prime}$ in Eq. (23) are straightforward. From (24) to (25) we rewrote the argument of the Dirac delta distribution such that $\varphi^{\prime}$ appears only once, using the identity $\sin \varphi^{\prime} \sin \bar{\varphi}+\cos \varphi^{\prime} \cos \bar{\varphi}=\cos \left(\varphi^{\prime}-\bar{\varphi}\right)$.

Given the complexity of the overlap condition between two arbitrarily oriented platelets in three dimensions, it is not surprising that Eq. (26) is an involved expression. It can be viewed as counting the number of intersections between the platelet $D^{\prime}$ and the rim of platelet $D$ 56]. The second term in Eq. (22) counts the number of intersections between platelet $D$ and the rim of platelet $D^{\prime}$. Hence in total there are two (zero) intersections for an overlapping (nonoverlapping) configuration, and indeed the Mayer bond between platelets, Eq. (22), is recovered through convolution of (orientation-dependent) single-particle measures.

\section{The platelet-needle Mayer bond}

We next consider the platelet-needle Mayer bond, $f_{D N}\left(\mathbf{r}, \Omega_{D} ; \boldsymbol{\Omega}_{N}\right)$, where $\mathbf{r}$ is the differ-

ence vector from disk center to needle center, and $\Omega_{D}$ and $\Omega_{N}$ are the orientations of the 


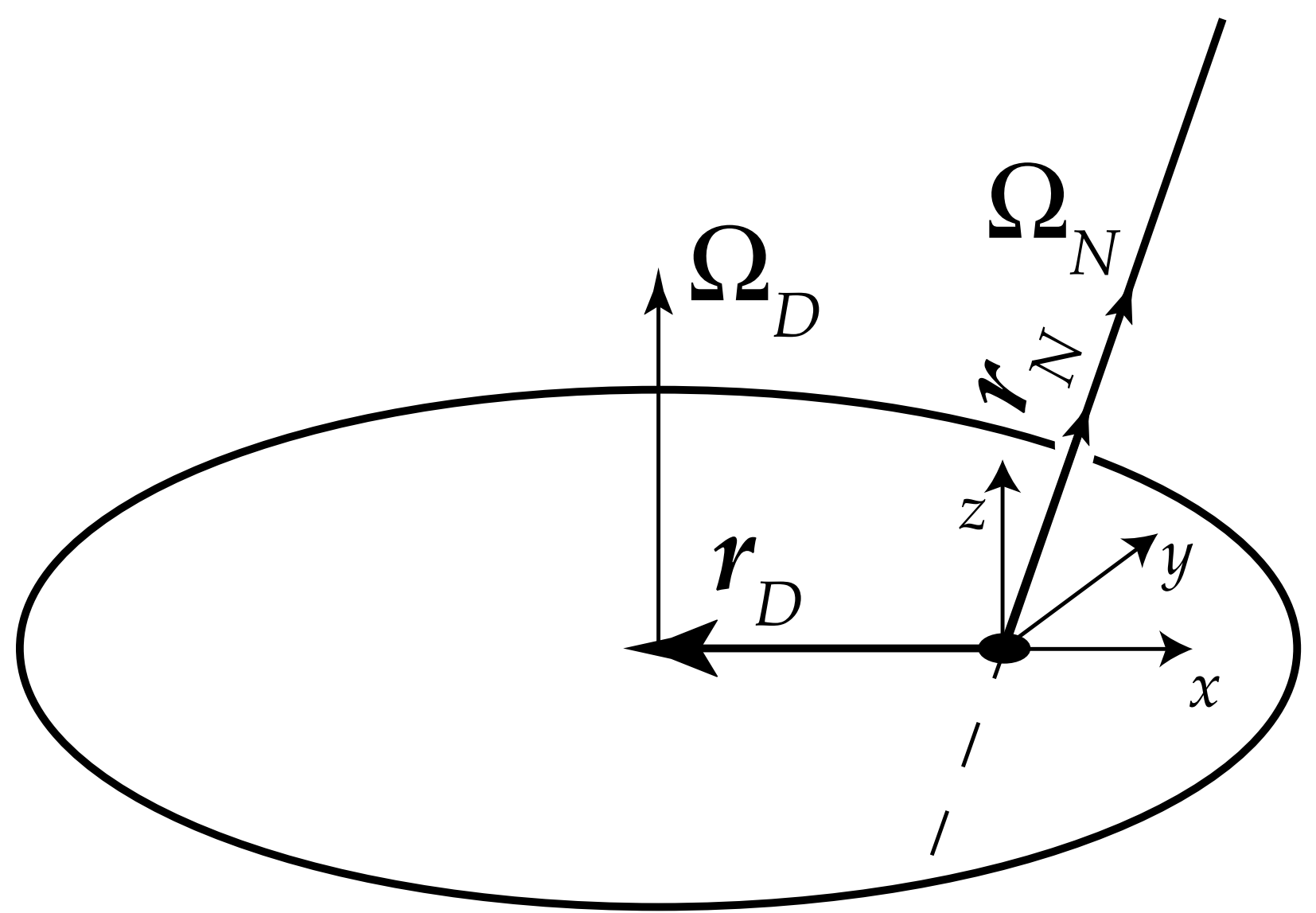

FIG. 4: Setup for the calculation of the platelet-needle Mayer bond. The needle is located at $\mathbf{r}_{N}$ in such a way that it points at the origin. The platelet is located at $\mathbf{r}_{D}$.

disk and needle, respectively. We define a "mixed" weight function for the platelets that is non-vanishing on the platelet surface, but carries an additional dependence on the rod orientation,

$$
w_{2}^{D N}\left(\mathbf{r}, \boldsymbol{\Omega}_{D} ; \boldsymbol{\Omega}_{N}\right)=2\left|\boldsymbol{\Omega}_{D} \cdot \boldsymbol{\Omega}_{N}\right| w_{2}^{D}\left(\mathbf{r}, \boldsymbol{\Omega}_{D}\right),
$$

where $w_{2}^{D}(\mathbf{r}, \boldsymbol{\Omega})$, as given in Eq. (18), describes the platelet surface. This allows us to obtain the Mayer bond between platelet and needle via

$$
-f_{D N}\left(\mathbf{r}, \boldsymbol{\Omega}_{D} ; \boldsymbol{\Omega}_{N}\right)=w_{2}^{D N}\left(\mathbf{r}, \boldsymbol{\Omega}_{D} ; \boldsymbol{\Omega}_{N}\right) * w_{1}^{N}\left(\mathbf{r}, \boldsymbol{\Omega}_{N}\right) .
$$

The validity of Eq. (28) can be seen by employing cylindrical coordinates and putting the platelet on the $y$-axis, its orientation being along the $z$-axis. Position the needle in such a way that its intersection with the $x-y$ plane is the origin (cf. Fig. (4). The needle is described by $\mathbf{r}_{N}=\left(z_{N} / \bar{z} \sqrt{1-\bar{z}^{2}} \sin \bar{\varphi}, z_{N} / \bar{z} \sqrt{1-\bar{z}^{2}} \cos \bar{\varphi}, z_{N}\right)$ and $\boldsymbol{\Omega}_{N}=$ $\left(\sqrt{1-\bar{z}^{2}} \sin \bar{\varphi}, \sqrt{1-\bar{z}^{2}} \cos \bar{\varphi}, \bar{z}\right)$. The coordinates of the platelet are $\mathbf{r}_{D}=\left(0, r_{D}, 0\right)$ and 
$\boldsymbol{\Omega}_{D}=(0,0,1)$; the integration variable is $\mathbf{x}=\left(x^{\prime} \sin \varphi^{\prime}, x^{\prime} \cos \varphi^{\prime}, z^{\prime}\right)$. From (27) and (18) we obtain the explicit expression $w_{2}^{D N}\left(\mathbf{r}, \boldsymbol{\Omega}_{D} ; \boldsymbol{\Omega}_{N}\right)=4 \Theta\left(R_{D}-|\mathbf{r}|\right) \delta\left(\mathbf{r} \cdot \boldsymbol{\Omega}_{D}\right)\left|\boldsymbol{\Omega}_{D} \cdot \boldsymbol{\Omega}_{N}\right|$, that we insert together with $w_{1}^{N}\left(\mathbf{r}, \boldsymbol{\Omega}_{N}\right)$, as given in Eq. (66), into Eq. (28) to obtain

$$
\begin{aligned}
&-f_{D N}\left(\mathbf{r}, \Omega_{D} ; \boldsymbol{\Omega}_{N}\right)=\int \mathrm{d}^{3} x w_{1}^{N}\left(\mathbf{x}-\mathbf{r}_{N}, \Omega_{N}\right) w_{2}^{D N}\left(\mathbf{x}-\mathbf{r}_{D}, \Omega_{D} ; \Omega_{N}\right) \\
&=\int_{-\infty}^{\infty} \mathrm{d} z^{\prime} \int_{0}^{2 \pi} \mathrm{d} \varphi^{\prime} \int_{0}^{\infty} \mathrm{d} x^{\prime} x^{\prime} \int_{-L / 2}^{L / 2} \mathrm{~d} l \\
& \times \delta\left(x^{\prime} \sin \varphi^{\prime}-z_{N} / \bar{z} \sqrt{1-\bar{z}^{2}} \sin \bar{\varphi}+l \sqrt{1-\bar{z}^{2}} \sin \bar{\varphi}\right) \\
& \times \delta\left(x^{\prime} \cos \varphi^{\prime}-z_{N} / \bar{z} \sqrt{1-\bar{z}^{2}} \cos \bar{\varphi}+l \sqrt{1-\bar{z}^{2}} \cos \bar{\varphi}\right) \\
& \times \delta\left(z^{\prime}-z_{N}+l \bar{z}\right) \Theta\left(R_{D}-\left|\left(x^{\prime} \sin \varphi^{\prime}, x^{\prime} \cos \varphi^{\prime}-r_{D}, z^{\prime}\right)\right|\right)|\bar{z}| \delta\left(z^{\prime}\right) \\
&= \int_{0}^{2 \pi} \mathrm{d} \varphi^{\prime} \int_{0}^{\infty} \mathrm{d} x^{\prime} x^{\prime} \delta\left(x^{\prime} \sin \varphi^{\prime}\right) \delta\left(x^{\prime} \cos \varphi^{\prime}\right) \\
& \times \Theta\left(R_{D}-\left|\left(x^{\prime} \sin \varphi^{\prime}, x^{\prime} \cos \varphi^{\prime}-r_{D}\right)\right|\right) \Theta\left(\frac{L}{2}|\bar{z}|-\left|z_{N}\right|\right) \\
&= \Theta\left(R_{D}-r_{D}\right) \Theta\left(\frac{L}{2}|\bar{z}|-\left|z_{N}\right|\right),
\end{aligned}
$$

where the integrals over $z^{\prime}$ and $l$ are straightforward to calculate. In Eq. (31), the integral over $\varphi^{\prime}$ is performed first, rendering the $x^{\prime}$-integral trivial.

The result, Eq. (32), is indeed the Mayer bond between platelet and needle: Due to the set-up, an overlap can occur only at the origin and the step functions in Eq. (32) provide a means to assess whether or not this is the case. The first step function checks whether the origin is inside the platelet while the second step function checks whether the needle intersects the origin.

\section{E. The sphere-platelet Mayer bond}

Treating the sphere-platelet Mayer bond leads to the most involved geometry of pairwise particle overlap in the ternary mixture under consideration. It turns out that this case is intimately related to the Mayer bond of the strictly two-dimensional hard disk mixtures. As detailed in Sec. [IIB the FMT gives only an approximate representation in this case. Still the Rosenfeld functional for two-dimensional hard disks is a reasonably accurate theory, see e.g. 45] for a recent study. Here we deal with this problem on the same level of approximation as the two-dimensional Rosenfeld case. Note that cutting the sphere with the plane of the 
platelet yields a disk as the shape of intersection. We need to consider its overlap with a platelet, and introduce the weight functions

$$
\begin{aligned}
w_{2}^{S D}(\mathbf{r}, \boldsymbol{\Omega}) & =\frac{4}{\pi} \sqrt{1-(\mathbf{r} \cdot \boldsymbol{\Omega} / R)^{2}} w_{2}^{S}(\mathbf{r}) \\
\mathbf{w}_{v 2}^{S D}(\mathbf{r}, \boldsymbol{\Omega}) & =4 \frac{\mathbf{r}-(\mathbf{r} \cdot \boldsymbol{\Omega}) \boldsymbol{\Omega}}{\pi R} w_{2}^{S}(\mathbf{r}) \\
\mathbf{w}_{v 1}^{D}(\mathbf{r}, \boldsymbol{\Omega}) & =\frac{\mathbf{r}}{R_{D}} w_{1}^{D}(\mathbf{r}, \boldsymbol{\Omega})
\end{aligned}
$$

where $w_{2}^{S}(\mathbf{r})$, as given in Eq. (2), describes the sphere surface, and $w_{1}^{D}(\mathbf{r}, \Omega)$, as given in Eq. (19), describes the platelet rim. Eq. (34) defines a vector field tied to the surface of the sphere (with radius $R$ ). In contrast to the "radial hedgehog" of the (classic) vector weight function $\mathbf{w}_{v 2}^{S}(\mathbf{r})$, as given in Eq. (3) , the direction of the current vector field is radial with respect to the (platelet) $\Omega$ direction and its magnitude decreases towards either pole - a configuration one could refer to as a "cylindrical hedgehog". Note further that $w_{2}^{S D}=\left|\mathbf{w}_{v 2}^{S D}\right|$, in accordance with $w_{2}^{S}=\left|\mathbf{w}_{v 2}^{S}\right|$. The vector-field of Eq. (35) resembles a corona and is the straightforward generalization of the corresponding two-dimensional hard disk weight function, $\mathbf{w}_{v 1}^{(i)}(\mathbf{r})$, given in Eq. (11). We use these functions to approximate $f_{S D} \approx f_{S D}^{*}$ with

$$
\begin{aligned}
-f_{S D}^{*}(\mathbf{r}, \boldsymbol{\Omega})= & w_{3}^{S}(\mathbf{r}) * w_{0}^{D}(\mathbf{r}, \boldsymbol{\Omega})+w_{1}^{S}(\mathbf{r}) * w_{2}^{D}(\mathbf{r}, \boldsymbol{\Omega}) \\
& +w_{2}^{S D}(\mathbf{r}, \boldsymbol{\Omega}) * w_{1}^{D}(\mathbf{r}, \boldsymbol{\Omega})-\mathbf{w}_{v 2}^{S D}(\mathbf{x}, \boldsymbol{\Omega}) * \mathbf{w}_{v 1}^{D}(\mathbf{r}, \boldsymbol{\Omega}),
\end{aligned}
$$

where again the convolution implies a scalar product between vectors. The three terms on the r.h.s. of Eq. (36) represent the arc that the rim of the platelet traces inside the sphere, the arc that the sphere traces on the platelet (as illustrated in Figs. 5] and 6. respectively), and an additional contribution of the cusps where the two arcs meet. We put the platelet into the $x-y$ plane with its center at the origin. The center of the sphere is located in the $y-z$ plane, see Fig 5, the coordinates chosen are $\mathbf{r}=(0, r, z), \boldsymbol{\Omega}=(0,0,1), \mathbf{x}=\left(r^{\prime} \sin \varphi^{\prime}, r^{\prime} \cos \varphi^{\prime}, z^{\prime}\right)$. We obtain for the first term on the r.h.s. of Eq. (36)

$$
\begin{aligned}
\int \mathrm{d}^{3} x w_{0}^{D}(\mathbf{x}, \boldsymbol{\Omega}) w_{3}^{S}(\mathbf{x}-\mathbf{r}) & \\
= & \frac{1}{2 \pi R_{D}} \int_{0}^{2 \pi} \mathrm{d} \varphi^{\prime} \int_{-\infty}^{\infty} \mathrm{d} z^{\prime} \int_{0}^{\infty} r^{\prime} \mathrm{d} r^{\prime} \delta\left(R_{D}-\sqrt{r^{\prime 2}+z^{\prime 2}}\right) \delta(\mathbf{x} \cdot \boldsymbol{\Omega}) \\
& \quad \times \Theta(R-|\mathbf{x}-\mathbf{r}|) \\
= & \frac{1}{\pi} \arccos \left(\frac{r^{2}+R_{D}^{2}+z^{2}-R^{2}}{2 r R_{D}}\right) \Theta\left(2 r R_{D}-\left|r^{2}+R_{D}^{2}+z^{2}-R^{2}\right|\right) .
\end{aligned}
$$




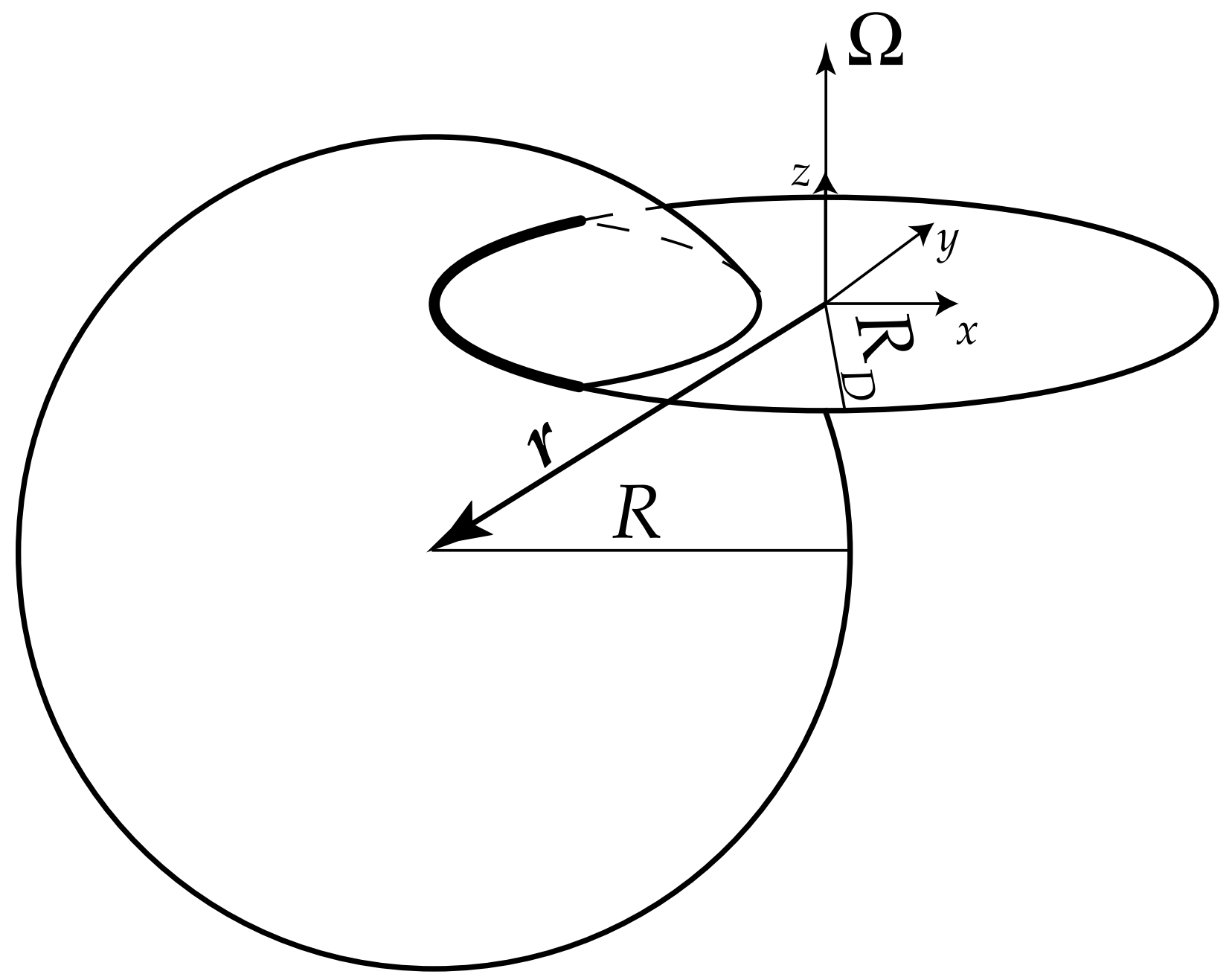

FIG. 5: Setup for the calculation of the sphere-platelet Mayer bond. The platelet is located at the origin. The convolution $w_{0}^{D} * w_{3}^{S}$ is equal to the angle under which the arc shown in bold appears.

By the cosine theorem, this is equal (up to a factor of $2 \pi$ ) to the length of the arc which the rim of the disc traces inside the sphere, see Eq. (14). Note also that $R^{2}-z^{2}$ is the squared radius of the circle that the platelet cuts out of the sphere. 


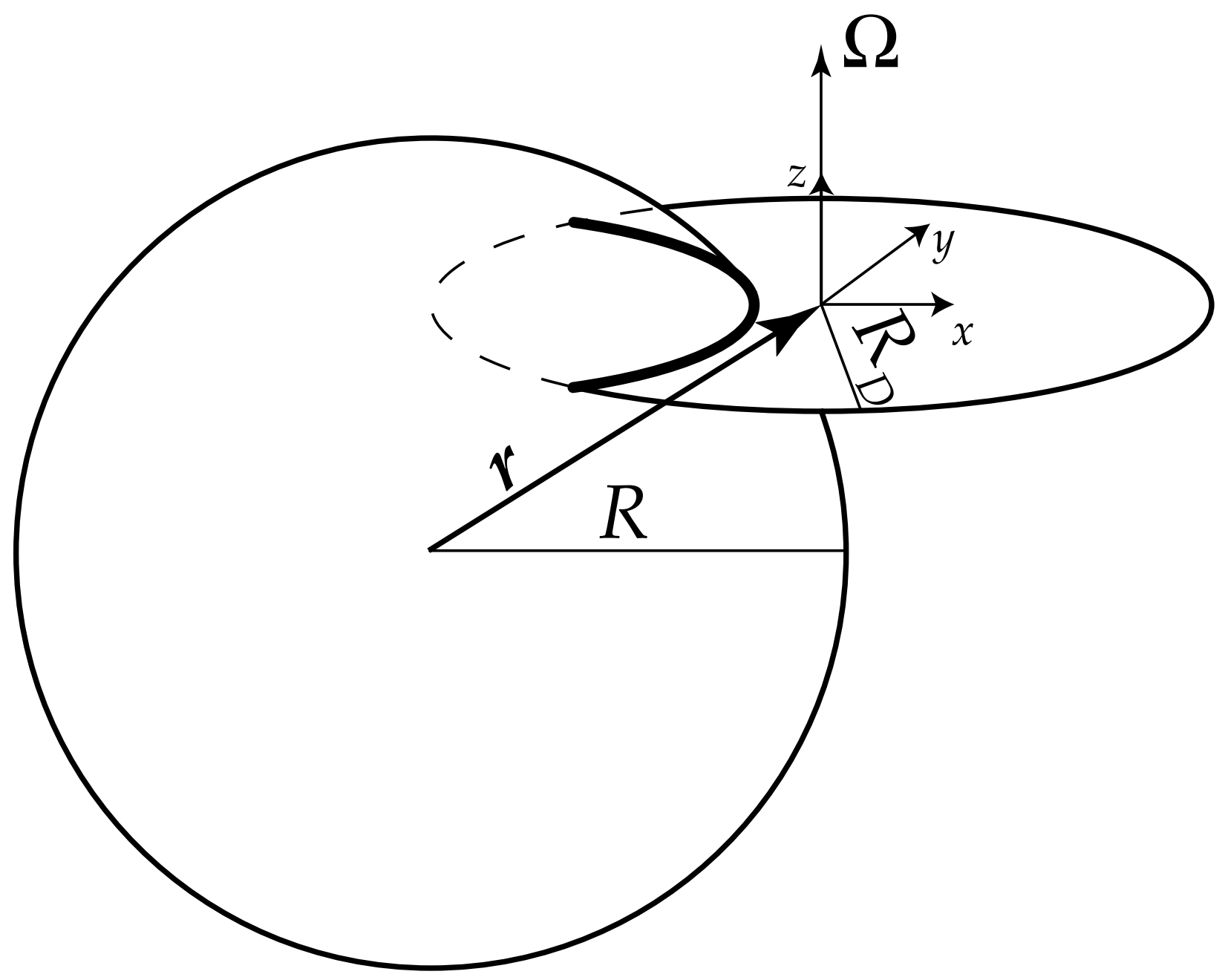

FIG. 6: Setup for the calculation of the sphere-platelet Mayer bond. The platelet is located at the origin. The convolution $w_{1}^{S} * w_{2}^{D}$ is equal to the angle under which the arc shown in bold appears.

For the second term on the r.h.s. of Eq. (36) we obtain

$$
\begin{aligned}
\int \mathrm{d}^{3} x w_{1}^{S}(\mathbf{x}) w_{2}^{D}(\mathbf{x}-\mathbf{r}, \Omega) & \\
= & \frac{1}{4 \pi R} \int_{-\infty}^{\infty} \mathrm{d} z^{\prime} \int_{0}^{2 \pi} \mathrm{d} \varphi^{\prime} \int_{0}^{\infty} \mathrm{d} r^{\prime} r^{\prime} \delta\left(R-\sqrt{r^{\prime 2}+z^{\prime 2}}\right) \\
& \times 2 \Theta\left(R_{D}-\sqrt{r^{2}+r^{\prime 2}+\left(z^{\prime}-z\right)^{2}-2 r r^{\prime} \cos \varphi^{\prime}}\right) \delta\left(z^{\prime}-z\right) \\
= & \frac{1}{2 \pi} \int_{0}^{2 \pi} \mathrm{d} \varphi^{\prime} \Theta(R-|z|) \Theta\left(R_{D}-\sqrt{r^{2}+R^{2}-z^{2}-2 r \sqrt{R^{2}-z^{2}} \cos \varphi^{\prime}}\right) \\
= & \frac{1}{\pi} \arccos \left(\frac{r^{2}+R^{2}-z^{2}-R_{D}^{2}}{2 r \sqrt{R^{2}-z^{2}}}\right) \Theta\left(2 r \sqrt{R^{2}-z^{2}}-\left|r^{2}+R^{2}-z^{2}-R_{D}^{2}\right|\right),
\end{aligned}
$$

which is indeed the length of the arc that the sphere traces on the platelet divided by $2 \pi$, see Eq (14). 
The relevant weight functions to treat the third and the fourth term on the r.h.s. of Eq. (36) are explicitly $\mathbf{w}_{v 2}^{S D}(\mathbf{r}, \boldsymbol{\Omega})=4 \delta(R-|\mathbf{r}|)[\mathbf{r}-(\mathbf{r} \cdot \boldsymbol{\Omega}) \boldsymbol{\Omega}] /(\pi R)$ and $\mathbf{w}_{v 1}^{D}(\mathbf{r}, \boldsymbol{\Omega})=$ $\delta\left(R_{D}-|\mathbf{r}|\right) \delta(\mathbf{r} \cdot \boldsymbol{\Omega}) \mathbf{r} /\left(8 R_{D}\right)$, hence

$$
\begin{aligned}
& \int \mathrm{d}^{3} x\left[w_{2}^{S D}(\mathbf{x}) w_{1}^{D}(\mathbf{x}-\mathbf{r})-\mathbf{w}_{v 2}^{S D}(\mathbf{x}) \mathbf{w}_{v 1}^{D}(\mathbf{x}-\mathbf{r})\right] \\
& =\frac{1}{2 \pi} \int_{0}^{2 \pi} \mathrm{d} \varphi^{\prime} \int_{0}^{\infty} \mathrm{d} r^{\prime} r^{\prime} \int_{-\infty}^{\infty} \mathrm{d} z^{\prime} \delta\left(R-\sqrt{r^{\prime 2}+z^{\prime 2}}\right) \\
& \times \delta\left(R_{D}-\sqrt{r^{2}+r^{\prime 2}-2 r r^{\prime} \cos \varphi^{\prime}+\left(z^{\prime}-z\right)^{2}}\right) \\
& \times \delta\left(z^{\prime}-z\right)\left(\frac{r^{\prime}}{\sqrt{r^{\prime 2}+z^{\prime 2}}}-\frac{\left(\begin{array}{c}
r^{\prime} \sin \varphi^{\prime} \\
r^{\prime} \cos \varphi^{\prime} \\
0
\end{array}\right) \cdot\left(\begin{array}{c}
r^{\prime} \sin \varphi^{\prime} \\
r^{\prime} \cos \varphi^{\prime}-r \\
z^{\prime}-z
\end{array}\right)}{R_{D} \sqrt{r^{\prime 2}+z^{\prime 2}}}\right) \\
& =\frac{1}{2 \pi} \int_{0}^{2 \pi} \mathrm{d} \varphi^{\prime} \int_{0}^{\infty} r^{\prime} \mathrm{d} r^{\prime} \delta\left(R-\sqrt{r^{\prime 2}+z^{2}}\right) \\
& \times \delta\left(R_{D}-\sqrt{r^{2}+r^{\prime 2}-2 r r^{\prime} \cos \varphi^{\prime}}\right)\left(\frac{r^{\prime}}{\sqrt{r^{\prime 2}+z^{2}}}-\frac{r^{\prime 2}-r r^{\prime} \cos \varphi^{\prime}}{R_{D} \sqrt{r^{\prime 2}+z^{2}}}\right) \\
& =\frac{R}{2 \pi} \int_{0}^{2 \pi} \mathrm{d} \varphi^{\prime} \delta\left(R_{D}-\sqrt{r^{2}+R^{2}-z^{2}-2 r \sqrt{R^{2}-z^{2}} \cos \varphi^{\prime}}\right) \\
& \times \frac{\sqrt{R^{2}-z^{2}}}{R}\left(1-\frac{\sqrt{R^{2}-z^{2}}-r \cos \varphi^{\prime}}{R_{D}}\right) \Theta(R-|z|) \\
& =\frac{1}{\pi} \Theta\left(2 r \sqrt{R^{2}-z^{2}}-\left|r^{2}+R^{2}-z^{2}-R_{D}^{2}\right|\right) \Theta(R-|z|) \\
& \times \frac{R^{2}-\left(\sqrt{R^{2}-z^{2}}-R_{D}\right)^{2}}{\sqrt{4 r^{2}\left(R^{2}-z^{2}\right)-\left(r^{2}+R^{2}-z^{2}-R_{D}^{2}\right)^{2}}} \\
& =\frac{1}{\pi} \Theta\left(R_{D}-\left|R_{D}^{\prime}-r\right|\right) \frac{r^{2}-\left(R_{D}^{\prime}-R_{D}\right)^{2}}{\sqrt{4 r^{2} R_{D}^{\prime 2}-\left(r^{2}+R_{D}^{\prime 2}-R_{D}^{2}\right)^{2}}} \text {. }
\end{aligned}
$$

Considering again that $R_{D}^{\prime 2}=R^{2}-z^{2}$ is the squared radius of the circle that the plane of the platelet cuts out of the sphere, we recover Rosenfeld's approximation for the Mayer bond of hard disks in two dimensions, see Eq. (16). Hence the same defect, as displayed in Fig. 2 for two-dimensional hard disks, is encountered for the overlap between sphere and platelet. Based on the very reasonable quality of the two-dimensional Rosenfeld functional, we expect this to do little harm in typical applications of the theory. 


\section{THIRD VIRIAL LEVEL: TRIANGLE DIAGRAMS}

The contribution of third order in densities to the excess free energy of a mixture has the generic exact form of a sum of triangle diagrams,

$$
-\frac{1}{6} \sum_{i j k} \int \mathrm{d} \mathbf{s} \rho_{i}(\mathbf{s}) \int \mathrm{d} \mathbf{s}^{\prime} \rho_{j}\left(\mathbf{s}^{\prime}\right) \int \mathrm{d} \mathbf{s}^{\prime \prime} \rho_{k}\left(\mathbf{s}^{\prime \prime}\right) f_{i j}\left(\mathbf{s}, \mathbf{s}^{\prime}\right) f_{j k}\left(\mathbf{s}^{\prime}, \mathbf{s}^{\prime \prime}\right) f_{i k}\left(\mathbf{s}, \mathbf{s}^{\prime \prime}\right),
$$

where $i, j, k$ label the species, the sum runs over all species, $f_{i j}\left(\mathbf{s}, \mathbf{s}^{\prime}\right)$ is the Mayer bond between particles of species $i$ and $j$ with coordinates $\mathbf{s}$ and $\mathbf{s}^{\prime}$, respectively, and $\mathbf{s}$ (and its primed versions) is a composite variable appropriate for the species under consideration, i.e. for the case of (uniaxial) rotators like platelets $\mathbf{s}=(\mathbf{r}, \boldsymbol{\Omega}), \int \mathrm{d} \mathbf{s}=\int \mathrm{d} \mathbf{r} \int \mathrm{d} \boldsymbol{\Omega} /(4 \pi)$, and for spheres simply $\mathbf{s}=\mathbf{r}, \int \mathrm{d} \mathbf{s}=\int \mathrm{d} \mathbf{r}$.

Even for one-component hard spheres FMT yields only an approximation to (47) due to the occurrence of "lost cases" [46, 47]: The FMT approximation for the triple product of Mayer bonds in (47) is non-vanishing only for configurations $\mathbf{r}, \mathbf{r}^{\prime}$, and $\mathbf{r}^{\prime \prime}$, such that three spheres with (hard sphere) radius $R$ centered at these positions share a non-vanishing common volume of intersection. Note that the existence of such triple intersection implies pairwise intersections, but not vice versa: An illustrative example is that constituted by three touching spheres; see e.g. [46] for an in-depth discussion. The lost cases are compensated by overcounting the cases with triple intersection, such that a reasonable (exact in the pure hard sphere case) third virial coefficient for the bulk fluid results. The upshot is that new weight functions result, that can be, via weighted densities, incorporated to improve the accuracy of the density functional approximation.

Here we follow a similar strategy and aim at controlling the dependence on positions and orientations through appropriately constructed weight functions. For triplets of platelets in configurations with common triple intersection we can express

$$
\begin{aligned}
f_{D D}\left(\mathbf{r}-\mathbf{r}^{\prime}, \boldsymbol{\Omega} ; \boldsymbol{\Omega}^{\prime}\right) f_{D D}\left(\mathbf{r}-\mathbf{r}^{\prime \prime}, \boldsymbol{\Omega} ; \boldsymbol{\Omega}^{\prime \prime}\right) f_{D D}\left(\mathbf{r}^{\prime}-\mathbf{r}^{\prime \prime}, \boldsymbol{\Omega}^{\prime}, \boldsymbol{\Omega}^{\prime \prime}\right) \\
\quad=A_{D D D} \int \mathrm{d} \mathbf{x} w_{2}^{D D D}\left(\mathbf{r}-\mathbf{x}, \boldsymbol{\Omega} ; \boldsymbol{\Omega}^{\prime} ; \boldsymbol{\Omega}^{\prime \prime}\right) w_{2}^{D}\left(\mathbf{r}^{\prime}-\mathbf{x}, \boldsymbol{\Omega}^{\prime}\right) w_{2}^{D}\left(\mathbf{r}^{\prime \prime}-\mathbf{x}, \boldsymbol{\Omega}^{\prime \prime}\right),
\end{aligned}
$$

where $A_{D D D}$ is a constant, $w_{2}^{D}(\mathbf{r}, \boldsymbol{\Omega})$ is the surface weight function defined in Eq. (18), and we introduce an additional surface weight that carries a dependence on two further (platelet) orientations,

$$
w_{2}^{D D D}\left(\mathbf{r}, \boldsymbol{\Omega} ; \boldsymbol{\Omega}^{\prime} ; \boldsymbol{\Omega}^{\prime \prime}\right)=\frac{8}{\pi}\left|\boldsymbol{\Omega} \cdot\left(\boldsymbol{\Omega}^{\prime} \times \boldsymbol{\Omega}^{\prime \prime}\right)\right| w_{2}^{D}(\mathbf{r}, \boldsymbol{\Omega}),
$$


with normalization such that $\int \mathrm{d} \mathbf{r} \int \frac{d \boldsymbol{\Omega}}{4 \pi} \int \frac{d \boldsymbol{\Omega}^{\prime}}{4 \pi} \int \frac{d \boldsymbol{\Omega}^{\prime \prime}}{4 \pi} w_{2}^{D D D}\left(\mathbf{r}, \boldsymbol{\Omega} ; \boldsymbol{\Omega}^{\prime} ; \boldsymbol{\Omega}^{\prime \prime}\right)=\xi_{2}^{D}=2 \pi R_{D}^{2}$. Setting $A_{D D D}=\pi / 64=0.0491$ yields equality in Eq. (48) for particle configurations with common triple intersection of the three particles shapes. Considering the bulk equation of state for pure platelets from SPT, and in particular its (approximative) third virial coefficient, the compensation for the lost cases (those without common triple intersection, but with pairwise intersections) results in $A_{D D D}=\pi / 32=0.0982$. In the following we will rather use the value from FMT, $A_{D D D}=1 /(4 \pi)=0.0796$, which is numerically similar to the SPT result. The advantage of the FMT scheme is that it consistently applies to mixtures, see Ref. [50] for a detailed discussion.

For the platelet-sphere mixture the triangle diagram stemming from a pair of platelets and a single sphere, provided that there is a a common intersection of all three particle shapes, can be expressed as

$$
\begin{aligned}
& f_{S D}\left(\mathbf{r} ; \mathbf{r}^{\prime}, \Omega^{\prime}\right) f_{D D}\left(\mathbf{r}^{\prime}, \Omega^{\prime} ; \mathbf{r}^{\prime \prime}, \Omega^{\prime \prime}\right) f_{S D}\left(\mathbf{r} ; \mathbf{r}^{\prime \prime}, \mathbf{\Omega}^{\prime \prime}\right) \\
& =\int \mathrm{d} \mathbf{x}\left[A_{S D D} w_{2}^{S D D}\left(\mathbf{r}-\mathbf{x} ; \boldsymbol{\Omega}^{\prime} ; \mathbf{\Omega}^{\prime \prime}\right) w_{2}^{D}\left(\mathbf{r}^{\prime}-\mathbf{x}, \boldsymbol{\Omega}^{\prime}\right) w_{2}^{D}\left(\mathbf{r}^{\prime \prime}-\mathbf{x}, \mathbf{\Omega}^{\prime \prime}\right)\right. \\
& +w_{3}^{S}(\mathbf{r}-\mathbf{x})\left(w_{1}^{D D}\left(\mathbf{r}^{\prime}-\mathbf{x}, \boldsymbol{\Omega}^{\prime} ; \boldsymbol{\Omega}^{\prime \prime}\right) w_{2}^{D}\left(\mathbf{r}^{\prime \prime}-\mathbf{x}, \boldsymbol{\Omega}^{\prime \prime}\right)\right. \\
& \left.\left.+w_{2}^{D}\left(\mathbf{r}^{\prime}-\mathbf{x}, \boldsymbol{\Omega}^{\prime}\right) w_{1}^{D D}\left(\mathbf{r}^{\prime \prime}-\mathbf{x}, \boldsymbol{\Omega}^{\prime \prime}, \boldsymbol{\Omega}^{\prime}\right)\right)\right],
\end{aligned}
$$

where $A_{S D D}$ is a constant, and we have introduced a sphere weight function that depends on two platelet orientations,

$$
w_{2}^{S D D}\left(\mathbf{r} ; \boldsymbol{\Omega}^{\prime} ; \mathbf{\Omega}^{\prime \prime}\right)=\frac{8}{\pi}\left|\left(\boldsymbol{\Omega}^{\prime} \times \boldsymbol{\Omega}^{\prime \prime}\right) \cdot \mathbf{w}_{v 2}^{S}(\mathbf{r})\right|
$$

with the normalization $\int \mathrm{d} \mathbf{r} \int \frac{d \boldsymbol{\Omega}}{4 \pi} \int \frac{d \boldsymbol{\Omega}^{\prime}}{4 \pi} w_{2}^{S D D}\left(\mathbf{r} ; \boldsymbol{\Omega}^{\prime} ; \boldsymbol{\Omega}^{\prime \prime}\right)=\xi_{2}^{S}=4 \pi R^{2}$. Again $A_{S D D}=$ $\pi / 64=0.0491$ yields equality in Eq. (50) provided that there is common triple intersection. We will use the FMT compensation, $A_{S D D}=1 /(4 \pi)=0.0796$.

The remaining case is that of triplets consisting of a single platelet and a pair of spheres. This case involves similar geometry as the platelet-sphere overlap, and hence we do not aim at an exact treatment. Again in the spirit of the two-dimensional hard disk case (cf. Sec. 
(IIB), we use an approximation,

$$
\begin{aligned}
& f_{S S}\left(\mathbf{r}^{\prime}-\mathbf{r}^{\prime \prime}\right) f_{S D}\left(\mathbf{r}^{\prime} ; \mathbf{r}, \boldsymbol{\Omega}\right) f_{S D}\left(\mathbf{r}^{\prime \prime} ; \mathbf{r}, \boldsymbol{\Omega}\right) \\
& \approx \int \mathrm{d} \mathbf{x}\left[w_{3}^{S}\left(\mathbf{r}^{\prime}-\mathbf{x}\right) w_{3}^{S}\left(\mathbf{r}^{\prime \prime}-\mathbf{x}\right) w_{0}^{D}(\mathbf{r}-\mathbf{x}, \boldsymbol{\Omega})\right. \\
& \quad+w_{3}^{S}\left(\mathbf{r}^{\prime}-\mathbf{x}\right)\left(w_{2}^{S D}\left(\mathbf{r}^{\prime \prime}-\mathbf{x} ; \boldsymbol{\Omega}\right) w_{1}^{D}(\mathbf{r}-\mathbf{x}, \boldsymbol{\Omega})-\mathbf{w}_{v 2}^{S D}\left(\mathbf{r}^{\prime \prime}-\mathbf{x} ; \boldsymbol{\Omega}\right) \cdot \mathbf{w}_{v 1}^{D}(\mathbf{r}-\mathbf{x}, \boldsymbol{\Omega})\right) \\
& \quad+w_{3}^{S}\left(\mathbf{r}^{\prime \prime}-\mathbf{x}\right)\left(w_{2}^{S D}\left(\mathbf{r}^{\prime}-\mathbf{x} ; \boldsymbol{\Omega}\right) w_{1}^{D}(\mathbf{r}-\mathbf{x}, \boldsymbol{\Omega})-\mathbf{w}_{v 2}^{S D}\left(\mathbf{r}^{\prime}-\mathbf{x} ; \boldsymbol{\Omega}\right) \cdot \mathbf{w}_{v 1}^{D}(\mathbf{r}-\mathbf{x}, \boldsymbol{\Omega})\right) \\
& \quad+A_{S S D} w_{2}^{D}(\mathbf{r}-\mathbf{x}, \boldsymbol{\Omega})\left(w_{2}^{S D}\left(\mathbf{r}^{\prime}-\mathbf{x} ; \boldsymbol{\Omega}\right) w_{2}^{S D}\left(\mathbf{r}^{\prime \prime}-\mathbf{x}, \boldsymbol{\Omega}\right)\right. \\
& \left.\left.\quad-\mathbf{w}_{v 2}^{S D}\left(\mathbf{r}^{\prime \prime}-\mathbf{x} ; \boldsymbol{\Omega}\right) \cdot \mathbf{w}_{v 2}^{S D}\left(\mathbf{r}^{\prime \prime}-\mathbf{x} ; \boldsymbol{\Omega}\right)\right)\right]
\end{aligned}
$$

where $A_{S S D}=1 / 16$ generates the best possible approximation and $A_{S S D}=1 /(4 \pi)$ compensates for the lost cases in the FMT approximation.

\section{WEIGHTED DENSITIES}

We first summarize the novel weight functions that describe the platelet degrees of freedom in the ternary mixture. From the original Rosenfeld prescription we have obtained [see Eqs. (18)-(201) in Sec. IIC

$$
\begin{aligned}
& w_{2}^{D}(\mathbf{r}, \boldsymbol{\Omega})=2 \Theta\left(R_{D}-|\mathbf{r}|\right) \delta(\mathbf{r} \cdot \boldsymbol{\Omega}), \\
& w_{1}^{D}(\mathbf{r}, \boldsymbol{\Omega})=\delta\left(R_{D}-|\mathbf{r}|\right) \delta(\mathbf{r} \cdot \boldsymbol{\Omega}) / 8, \\
& w_{0}^{D}(\mathbf{r}, \boldsymbol{\Omega})=\frac{1}{2 \pi R_{D}} \delta\left(R_{D}-|\mathbf{r}|\right) \delta(\mathbf{r} \cdot \boldsymbol{\Omega}) .
\end{aligned}
$$

In order to treat platelet-platelet and platelet-needle overlap we have introduced [see Eq. (21) in Sec. IIIC and Eq. (27) in Sec. IIID

$$
\begin{aligned}
& w_{1}^{D D}\left(\mathbf{r}, \boldsymbol{\Omega} ; \boldsymbol{\Omega}^{\prime}\right)=\frac{2}{R_{D}}\left|\boldsymbol{\Omega} \cdot\left(\boldsymbol{\Omega}^{\prime} \times \mathbf{r}\right)\right| w_{1}^{D}(\mathbf{r}, \boldsymbol{\Omega}), \\
& w_{2}^{D N}\left(\mathbf{r}, \boldsymbol{\Omega}_{D} ; \boldsymbol{\Omega}_{N}\right)=2\left|\boldsymbol{\Omega}_{D} \cdot \boldsymbol{\Omega}_{N}\right| w_{2}^{D}\left(\mathbf{r}, \boldsymbol{\Omega}_{D}\right),
\end{aligned}
$$

respectively. To treat sphere-platelet overlap we use [see Eq. (33)-(35) in Sec. IIIE]

$$
\begin{aligned}
w_{2}^{S D}(\mathbf{r}, \boldsymbol{\Omega}) & =\frac{4}{\pi} \sqrt{1-(\mathbf{r} \cdot \boldsymbol{\Omega} / R)^{2}} w_{2}^{S}(\mathbf{r}), \\
\mathbf{w}_{v 2}^{S D}(\mathbf{r}, \boldsymbol{\Omega}) & =4 \frac{\mathbf{r}-(\mathbf{r} \cdot \boldsymbol{\Omega}) \boldsymbol{\Omega}}{\pi R} w_{2}^{S}(\mathbf{r}) \\
\mathbf{w}_{v 1}^{D}(\mathbf{r}, \boldsymbol{\Omega}) & =\frac{\mathbf{r}}{R_{D}} w_{1}^{D}(\mathbf{r}, \boldsymbol{\Omega})
\end{aligned}
$$


The consideration of the third order contributions to the excess free energy has led to [see Eqs. (49) and (51) in Sec. IV

$$
\begin{aligned}
w_{2}^{D D D}\left(\mathbf{r}, \boldsymbol{\Omega} ; \boldsymbol{\Omega}^{\prime} ; \boldsymbol{\Omega}^{\prime \prime}\right) & =\frac{8}{\pi}\left|\boldsymbol{\Omega} \cdot\left(\boldsymbol{\Omega}^{\prime} \times \boldsymbol{\Omega}^{\prime \prime}\right)\right| w_{2}^{D}(\mathbf{r}, \boldsymbol{\Omega}), \\
w_{2}^{S D D}\left(\mathbf{r} ; \boldsymbol{\Omega}^{\prime} ; \boldsymbol{\Omega}^{\prime \prime}\right) & =\frac{8}{\pi}\left|\left(\boldsymbol{\Omega}^{\prime} \times \boldsymbol{\Omega}^{\prime \prime}\right) \cdot \mathbf{w}_{v 2}^{S}(\mathbf{r})\right| .
\end{aligned}
$$

All weight functions are used to build weighted densities via convolutions with the bare density profiles. For hard spheres the scalar and vectorial weighted densities are respectively given by

$$
\begin{aligned}
& n_{\nu}^{S}(\mathbf{r})=w_{\nu}^{S}(\mathbf{r}) * \rho_{S}(\mathbf{r}), \quad \nu=0,1,2,3 \\
& \mathbf{n}_{\nu}^{S}(\mathbf{r})=\mathbf{w}_{\nu}^{S}(\mathbf{r}) * \rho_{S}(\mathbf{r}), \quad \nu=v 1, v 2
\end{aligned}
$$

where $w_{\nu}^{S}(\mathbf{r})$ and $\mathbf{w}_{\nu}^{S}(\mathbf{r})$ as given in and below Eqs. (11)-(3) are the classic Rosenfeld hard sphere functions. Adding non-spherical particles requires convolving the sphere density profile with orientation-dependent weight functions. The coupling to needles is accomplished via

$$
n_{2}^{S N}(\mathbf{r}, \boldsymbol{\Omega})=w_{2}^{S N}(\mathbf{r}, \boldsymbol{\Omega}) * \rho_{S}(\mathbf{r})
$$

where $w_{2}^{S N}(\mathbf{r}, \boldsymbol{\Omega})$ as given in Eq. (17) is a mixed sphere-needle function. Treating the platelets follows a similar outline,

$$
\begin{aligned}
n_{2}^{S D}(\mathbf{r}, \boldsymbol{\Omega}) & =w_{2}^{S D}(\mathbf{r}, \boldsymbol{\Omega}) * \rho_{S}(\mathbf{r}), \\
\mathbf{n}_{v 2}^{S D}(\mathbf{r}, \boldsymbol{\Omega}) & =\mathbf{w}_{v 2}^{S D}(\mathbf{r}, \boldsymbol{\Omega}) * \rho_{S}(\mathbf{r}), \\
n_{2}^{S D D}\left(\mathbf{r} ; \boldsymbol{\Omega} ; \boldsymbol{\Omega}^{\prime}\right) & =w_{2}^{S D D}\left(\mathbf{r} ; \boldsymbol{\Omega} ; \boldsymbol{\Omega}^{\prime}\right) * \rho_{S}(\mathbf{r}),
\end{aligned}
$$

with $w_{2}^{S D}(\mathbf{r}, \boldsymbol{\Omega})$ and $\mathbf{w}_{v 2}^{S D}(\mathbf{r}, \boldsymbol{\Omega})$ given in Eqs. (33) and (134), respectively, and $w_{2}^{S D D}\left(\mathbf{r} ; \mathbf{\Omega}^{\prime} ; \mathbf{\Omega}^{\prime \prime}\right)$ as given in Eq. (51).

For needles the pure weight functions are

$$
n_{\nu}^{N}(\mathbf{r}, \boldsymbol{\Omega})=w_{\nu}^{N}(\mathbf{r}, \boldsymbol{\Omega}) * \rho_{N}(\mathbf{r}, \boldsymbol{\Omega}), \quad \nu=0,1
$$

where $w_{0}^{N}(\mathbf{r}, \boldsymbol{\Omega})$ and $w_{1}^{N}(\mathbf{r}, \boldsymbol{\Omega})$ are given in Eqs. (5) and (6), respectively. In case one considers needle-needle interactions, additional weighted densities need to be taken into account [39]. 
The degrees of freedom of the platelets are modelled through the weighted densities

$$
\begin{aligned}
n_{\nu}^{D}(\mathbf{r}, \boldsymbol{\Omega}) & =w_{\nu}^{D}(\mathbf{r}, \boldsymbol{\Omega}) * \rho_{D}(\mathbf{r}, \boldsymbol{\Omega}), \quad \nu=0,1,2, \\
\mathbf{n}_{v 1}^{D}(\mathbf{r}, \boldsymbol{\Omega}) & =\mathbf{w}_{v 1}^{D}(\mathbf{r}, \boldsymbol{\Omega}) * \rho_{D}(\mathbf{r}, \boldsymbol{\Omega}), \\
n_{1}^{D D}\left(\mathbf{r}, \boldsymbol{\Omega}^{\prime}\right) & =\int \frac{\mathrm{d} \boldsymbol{\Omega}}{4 \pi} w_{1}^{D D}\left(\mathbf{r}, \boldsymbol{\Omega} ; \boldsymbol{\Omega}^{\prime}\right) * \rho_{D}(\mathbf{r}, \boldsymbol{\Omega}), \\
n_{2}^{D D D}\left(\mathbf{r} ; \boldsymbol{\Omega} ; \boldsymbol{\Omega}^{\prime}\right) & =\int \frac{\mathrm{d} \boldsymbol{\Omega}^{\prime \prime}}{4 \pi} w_{2}^{D D D}\left(\mathbf{r}, \boldsymbol{\Omega}^{\prime \prime} ; \boldsymbol{\Omega} ; \boldsymbol{\Omega}^{\prime}\right) * \rho_{D}\left(\mathbf{r}, \boldsymbol{\Omega}^{\prime \prime}\right),
\end{aligned}
$$

with the weight functions $w_{2}^{D}(\mathbf{r}, \boldsymbol{\Omega}), w_{1}^{D}(\mathbf{r}, \boldsymbol{\Omega})$, and $w_{0}^{D}(\mathbf{r}, \boldsymbol{\Omega})$, as given in Eqs. (18), (19), and (20), respectively, and $\mathbf{w}_{v 1}^{D}(\mathbf{r}, \boldsymbol{\Omega})$ as given in Eq. (35)). The double orientation-dependent weight function $w_{1}^{D D}\left(\mathbf{r}, \boldsymbol{\Omega} ; \boldsymbol{\Omega}^{\prime}\right)$ is given in Eq. (21). The weight function that we have introduced to control the triangle diagrams, $w_{2}^{D D D}\left(\mathbf{r}, \boldsymbol{\Omega}^{\prime} ; \boldsymbol{\Omega}^{\prime \prime} ; \boldsymbol{\Omega}^{\prime \prime \prime}\right)$, is given in Eq. (49).

The remaining coupling of the platelets to the needles is achieved via

$$
n_{2}^{D N}\left(\mathbf{r}, \boldsymbol{\Omega}^{\prime}\right)=\int \frac{\mathrm{d} \boldsymbol{\Omega}}{4 \pi} w_{2}^{D N}\left(\mathbf{r}, \boldsymbol{\Omega} ; \boldsymbol{\Omega}^{\prime}\right) * \rho_{D}(\mathbf{r}, \boldsymbol{\Omega}),
$$

where we have used $w_{2}^{D N}(\mathbf{r}, \boldsymbol{\Omega})$ as given in Eq. (27) to build a mixed weighted density.

\section{FREE ENERGY FUNCTIONAL}

In Ref. 38] the excess Helmholtz free energy functional for particles with rotational degrees of freedom was expressed not only as a spatial integral (as was proposed in Refs. 21, 22]), but also as an integral over the director space. In our present explicit consideration of three particle correlations an additional integration over director space is required,

$$
\beta F_{\mathrm{exc}}=\int \mathrm{d} \mathbf{r} \int \frac{\mathrm{d} \boldsymbol{\Omega}}{4 \pi} \int \frac{\mathrm{d} \boldsymbol{\Omega}^{\prime}}{4 \pi} \Phi\left(\left\{n_{\nu}^{i}\right\}\right)
$$

where the (reduced) free energy density, $\Phi$, is a function of the set of weighted densities, $\left\{n_{\nu}^{i}\right\}$, where $i$ labels the species and $\nu$ labels the type of weight function.

For hard spheres $\Phi=\Phi_{S}$ with

$$
\begin{aligned}
\Phi_{S}= & -n_{0}^{S} \ln \left(1-n_{3}^{S}\right)+\frac{n_{1}^{S} n_{2}^{S}-\mathbf{n}_{v 1}^{S} \cdot \mathbf{n}_{v 2}^{S}}{1-n_{3}^{S}} \\
& +\frac{\frac{1}{3}\left(n_{2}^{S}\right)^{3}-n_{2}^{S} \mathbf{n}_{v 2}^{S} \cdot \mathbf{n}_{v 2}^{S}}{8 \pi\left(1-n_{3}^{S}\right)^{2}}
\end{aligned}
$$

where we have suppressed here and in the following the dependence of the weighted densities on the space coordinate r. Eq. (76) is the original Rosenfeld form 20] that yields, for bulk 
fluids, the (reduced) free energy density as obtained by the Percus-Yevick compressibility (or scaled-particle) route. Improved versions exist that feature exact dimensional crossover [51, 52], and the Carnahan-Starling equation for bulk fluid states [53].

For binary mixtures of spheres and needles the reduced free energy density is $\Phi=\Phi_{S}+$ $\Phi_{S N}$, where $\Phi_{S}$ is given in (76), and $\Phi_{S N}$ describes the effect of needle-sphere interactions [38], given as

$$
\Phi_{S N}=-n_{0}^{N}(\boldsymbol{\Omega}) \ln \left(1-n_{3}^{S}\right)+\frac{n_{1}^{N}(\boldsymbol{\Omega}) n_{2}^{S N}(\boldsymbol{\Omega})}{1-n_{3}^{S}},
$$

where we suppress the dependence of the weighted densities on $\mathbf{r}$, but make the dependence on orientation explicit in the notation. The free energy for bulk fluid states obtained from (777) is the same as that obtained from a thermodynamic (free-volume-like) perturbation theory [37], that results in a fluid-fluid demixing binodal that compares well with results from simulations.

For a system of pure platelets $\Phi=\Phi_{D}$, with

$$
\Phi_{D}=n_{1}^{D D}(\boldsymbol{\Omega}) n_{2}^{D}(\boldsymbol{\Omega})+\frac{1}{24 \pi} n_{2}^{D}(\boldsymbol{\Omega}) n_{2}^{D D D}\left(\boldsymbol{\Omega}, \boldsymbol{\Omega}^{\prime}\right) n_{2}^{D}\left(\boldsymbol{\Omega}^{\prime}\right),
$$

where the prefactor of the third order term in density is according to Rosenfeld $A=$ $1 /(24 \pi)=0.01326$. Scaled-particle theory [50, 54] gives a slightly different value, namely $A=\pi / 192=0.01636$; see Ref. [50] for a detailed comparison of the predictions of these approaches for bulk fluid properties. Eq. (78) is the simplest form of a free energy density that features the correct second virial level and a similar free energy for bulk isotropic fluid states as scaled-particle theory.

For binary sphere-platelet mixtures we obtain $\Phi=\Phi_{S}+\Phi_{S D}$, where the hard sphere contribution $\Phi_{S}$ is given in Eq. (76)), and

$$
\begin{aligned}
\Phi_{S D}= & -n_{0}^{D}(\boldsymbol{\Omega}) \ln \left(1-n_{3}^{S}\right) \\
& +\frac{n_{1}^{S} n_{2}^{D}(\boldsymbol{\Omega})+n_{2}^{S D}(\boldsymbol{\Omega}) n_{1}^{D}(\boldsymbol{\Omega})-\mathbf{n}_{v 2}^{S D}(\boldsymbol{\Omega}) \cdot \mathbf{n}_{v 1}^{D}(\boldsymbol{\Omega})}{1-n_{3}^{S}}+\frac{n_{1}^{D D}(\boldsymbol{\Omega}) n_{2}^{D}(\boldsymbol{\Omega})}{1-n_{3}^{S}} \\
& +\frac{\left(n_{2}^{S D}(\boldsymbol{\Omega}) n_{2}^{S D}(\boldsymbol{\Omega})-\mathbf{n}_{v 2}^{S D}(\boldsymbol{\Omega}) \cdot \mathbf{n}_{v 2}^{S D}(\boldsymbol{\Omega})\right) n_{2}^{D}(\boldsymbol{\Omega})}{8 \pi\left(1-n_{3}^{S}\right)^{2}} \\
& +\frac{n_{2}^{S D D}\left(\boldsymbol{\Omega}, \boldsymbol{\Omega}^{\prime}\right) n_{2}^{D}(\boldsymbol{\Omega}) n_{2}^{D}\left(\boldsymbol{\Omega}^{\prime}\right)}{8 \pi\left(1-n_{3}^{S}\right)^{2}}+\frac{n_{2}^{D D D}\left(\boldsymbol{\Omega}, \boldsymbol{\Omega}^{\prime}\right) n_{2}^{D}(\boldsymbol{\Omega}) n_{2}^{D}\left(\boldsymbol{\Omega}^{\prime}\right)}{24 \pi\left(1-n_{3}^{S}\right)^{2}} .
\end{aligned}
$$

For a binary platelet-needle mixture the free energy density is $\Phi=\Phi_{D}+\Phi_{D N}$, where the pure platelet contribution $\Phi_{D}$ is given in Eq. (78), and a simple second-order coupling 
between both species is introduced via

$$
\Phi_{D N}=n_{2}^{D N}\left(\Omega ; \Omega^{\prime}\right) n_{1}^{N}\left(\boldsymbol{\Omega}^{\prime}\right)
$$

Due to the vanishing thickness of particles of both species, any higher order terms vanish according to SPT.

Finally, for ternary sphere-platelet-needle mixtures the free energy density is

$$
\Phi=\Phi_{S}+\Phi_{S N}+\Phi_{S D}+\frac{n_{2}^{D N}\left(\Omega ; \Omega^{\prime}\right) n_{1}^{N}\left(\Omega^{\prime}\right)}{1-n_{3}^{S}}
$$

where the first three terms on the r.h.s. are given through Eqs. (76), (177) and (79), respectively.

\section{PLANAR GEOMETRY}

In many practical situations one is faced with inhomogeneities that depend only on a single (Cartesian) coordinate, while being translationally invariant in the two remaining directions. A smooth planar wall, where the fluid density profile(s) only depend on the perpendicular distance, $z$, from the wall is a primary example. For rotators an additional simplification arises from (cylindrical) rotational symmetry around the $z$-axis. Hence such problems are characterized solely by $z$, and the tilt angle $\vartheta$ between the particle orientation and the $z$-axis. We give in the following explicit expressions for (reduced) weight functions appropriate for efficient numerical treatment of such situations. For completeness we also give the results for spheres and needles [39].

Let us start by defining the full set of reduced densities; we use the parametrizations $\mathbf{r}=(x, y, z)$ and $\boldsymbol{\Omega}=(\vartheta, \varphi)$. For the spheres

$$
w_{\nu}^{S}(z)=\int \mathrm{d} x \int \mathrm{d} y w_{\nu}^{S}(\mathbf{r}), \quad \nu=3,2,1,0, v 2, v 1
$$

Further mixed weight functions couple spheres to needles,

$$
w_{2}^{S N}(z, \vartheta)=\int \mathrm{d} x \int \mathrm{d} y w_{2}^{S N}(\mathbf{r}, \mathbf{\Omega})
$$


and spheres to platelets

$$
\begin{aligned}
w_{2}^{S D}(z, \vartheta) & =\int \mathrm{d} x \int \mathrm{d} y w_{2}^{S D}(\mathbf{r}, \boldsymbol{\Omega}), \\
\mathbf{w}_{v 2}^{S D}(z, \vartheta) & =\int \frac{\mathrm{d} \varphi}{2 \pi} \int \mathrm{d} x \int \mathrm{d} y \mathbf{w}_{v 2}^{S D}(\mathbf{r}, \boldsymbol{\Omega}), \\
w_{2}^{S D D}\left(z, \vartheta, \vartheta^{\prime}\right) & =\int \frac{\mathrm{d} \varphi}{2 \pi} \int \frac{\mathrm{d} \varphi^{\prime}}{2 \pi} \int \mathrm{d} x \int \mathrm{d} y w_{2}^{S D D}\left(\mathbf{r}, \boldsymbol{\Omega}, \mathbf{\Omega}^{\prime}\right),
\end{aligned}
$$

For the needles

$$
w_{\nu}^{N}(z, \vartheta)=\int \mathrm{d} x \int \mathrm{d} y w_{\nu}^{N}(\mathbf{r}, \boldsymbol{\Omega}), \quad \nu=0,1
$$

For the platelets the effective weight functions for planar geometry are obtained by integrating over the lateral coordinates

$$
w_{\nu}^{D}(z, \vartheta)=\int \mathrm{d} x \int \mathrm{d} y w_{\nu}^{D}(\mathbf{r}, \mathbf{\Omega}), \quad \nu=0,1,2,
$$

and

$$
\begin{aligned}
\mathbf{w}_{v 1}^{D}(z, \vartheta) & =\int \frac{\mathrm{d} \varphi}{2 \pi} \int \mathrm{d} x \int \mathrm{d} y \mathbf{w}_{v 1}^{D}(\mathbf{r}, \boldsymbol{\Omega}), \\
w_{1}^{D D}\left(z, \vartheta, \vartheta^{\prime}\right) & =\int \frac{\mathrm{d} \varphi^{\prime}}{2 \pi} \int \mathrm{d} x \int \mathrm{d} y w_{1}^{D D}\left(\mathbf{r}, \boldsymbol{\Omega}, \mathbf{\Omega}^{\prime}\right) \\
w_{2}^{D D D}\left(z, \vartheta, \vartheta^{\prime}, \vartheta^{\prime \prime}\right) & =\int \frac{\mathrm{d} \varphi^{\prime}}{2 \pi} \int \frac{\mathrm{d} \varphi^{\prime \prime}}{2 \pi} \int \mathrm{d} x \int \mathrm{d} y w_{2}^{D D D}\left(\mathbf{r}, \boldsymbol{\Omega}, \mathbf{\Omega}^{\prime}, \Omega^{\prime \prime}\right) \\
w_{2}^{D N}\left(z, \vartheta_{D} ; \vartheta_{N}\right) & =\int \frac{\mathrm{d} \varphi_{D}}{2 \pi} \int \mathrm{d} x \int \mathrm{d} y w_{2}^{D N}\left(\mathbf{r}, \Omega_{D} ; \Omega_{N}\right) .
\end{aligned}
$$

Using the effective weight functions the weighted densities can then be written as

$$
\begin{gathered}
\mathbf{n}_{v 2}^{S D}(z, \vartheta)=\mathbf{w}_{v 2}^{S D}(z, \vartheta) * \rho_{S}(z), \\
n_{\nu}^{D}(z, \vartheta)=w_{\nu}^{D}(z, \vartheta) * \rho_{D}(z, \vartheta), \quad \nu=0,1,2, \\
\mathbf{n}_{v 1}^{D}(z, \vartheta)=\mathbf{w}_{v 1}^{D}(z, \vartheta) * \rho_{D}(z, \vartheta), \\
n_{1}^{D D}\left(z, \vartheta^{\prime}\right)=\frac{1}{2} \int_{0}^{\pi} \mathrm{d} \vartheta \sin \vartheta w_{1}^{D D}\left(z, \vartheta ; \vartheta^{\prime}\right) * \rho_{D}(z, \vartheta), \\
n_{2}^{D D D}\left(z, \vartheta, \vartheta^{\prime}\right)=\frac{1}{2} \int_{0}^{\pi} \mathrm{d} \vartheta^{\prime \prime} w_{2}^{D D D}\left(z, \vartheta^{\prime \prime} ; \vartheta ; \vartheta^{\prime}\right) * \rho_{D}\left(z, \vartheta^{\prime \prime}\right) \\
n_{2}^{D N}\left(z, \vartheta^{\prime}\right)=\frac{1}{2} \int_{0}^{\pi} \mathrm{d} \vartheta \sin \vartheta w_{2}^{D N}\left(z, \vartheta ; \vartheta^{\prime}\right) * \rho_{D}(z, \vartheta),
\end{gathered}
$$


where in Eqs. (93)-98) (and only here) * denotes the one-dimensional convolution, $g(z) *$ $h(z)=\int g\left(z^{\prime}-z\right) h\left(z^{\prime}\right)$. In contrast to the needle case the integrands depend non-trivially on $\varphi$.

We finally give all results for the effective weight functions in planar geometry. For hard spheres the well-known results are

$$
\begin{aligned}
w_{3}^{S}(z) & =\pi\left(R^{2}-z^{2}\right) \Theta(R-|z|), \\
w_{2}^{S}(z) & =2 \pi R \Theta(R-|z|), \\
\mathbf{w}_{v 2}^{S}(z) & =2 \pi z \Theta(R-|z|) \mathbf{e}_{z},
\end{aligned}
$$

where $\mathbf{e}_{z}$ is the unit vector pointing along the $z$-axis. The linearly dependent weight functions are $w_{1}^{S}(z)=\Theta(R-|z|) / 2, w_{0}^{S}(z)=\Theta(R-|z|) /(2 R), \mathbf{w}_{v 1}^{S}(z)=z \Theta(R-|z|) \mathbf{e}_{z} /(2 R)$. The mixed sphere-needle weight function is given through

$$
w_{2}^{S N}(z, \vartheta)=\left\{\begin{array}{cl}
8 \sqrt{R^{2} \sin ^{2} \vartheta-z^{2}} \\
+8 z \cos \vartheta \\
\quad \times \arcsin \left(\frac{z \cot (\vartheta)}{\left.\sqrt{R^{2}-z^{2}}\right)}\right) & \text { if }|z|<R \sin \vartheta \\
4 \pi|z| \cos \vartheta & \text { if } R \sin \vartheta \leq|z| \leq R \\
0 & \text { otherwise. }
\end{array}\right.
$$

Further mixed sphere weight functions are

$$
\begin{aligned}
w_{2}^{S D}(z, \vartheta) & =\frac{2}{\pi^{2}} \Theta(R-|z|) \int_{0}^{2 \pi} d \varphi \sqrt{R^{2}-\left[\left(R^{2}-z^{2}\right) \cos \varphi \sin \vartheta+z \cos \vartheta\right]^{2}}, \\
\mathbf{w}_{v 2}^{S D}(z, \vartheta) & =\frac{8 \pi}{R_{D}} z \sin ^{2} \vartheta \sqrt{R_{D}^{2}-z^{2}} \Theta(R-|z|) \mathbf{e}_{z}, \\
w_{2}^{S D D}\left(z, \vartheta, \vartheta^{\prime}\right) & =\Theta(R-|z|) \int_{0}^{2 \pi} \mathrm{d} \varphi^{\prime \prime} \begin{cases}\frac{8}{\pi}|t| & \text { if } t^{2}>s^{2} \\
\frac{16}{\pi^{2}}\left(\sqrt{s^{2}-t^{2}}+t \arcsin \frac{t}{s}\right) & \text { else, }\end{cases}
\end{aligned}
$$

where $\mathbf{e}_{z}$ is a unit vector in the $z$-direction, $t=z \sin \vartheta \sin \vartheta^{\prime} \sin \varphi^{\prime}$, and $s=$ $\sqrt{R^{2}-z^{2}} \sqrt{\sin ^{2} \vartheta \cos ^{2} \vartheta^{\prime}+\cos ^{2} \vartheta \sin ^{2} \vartheta^{\prime}-2 \sin \vartheta \cos \vartheta \sin \vartheta^{\prime} \cos \vartheta^{\prime} \cos \varphi^{\prime}}$.

For the needles the effective weight functions are

$$
\begin{aligned}
& w_{1}^{N}(z, \vartheta)=(4 \cos \vartheta)^{-1} \Theta\left(\frac{L}{2} \cos \vartheta-|z|\right), \\
& w_{0}^{N}(z, \vartheta)=\frac{1}{2} \delta\left(\frac{L}{2} \cos \vartheta-|z|\right) .
\end{aligned}
$$


Carrying out the integrations in Eq. (88) yields

$$
\begin{aligned}
& w_{2}^{D}(z, \vartheta)=\frac{4 \sqrt{R_{D}^{2}-z^{2} / \sin ^{2} \vartheta}}{\sin \vartheta} \Theta\left(R_{D} \sin \vartheta-|z|\right) \\
& w_{1}^{D}(z, \vartheta)=\frac{R_{D} \Theta\left(R_{D} \sin \vartheta-|z|\right)}{4 \sqrt{R_{D}^{2} \sin \vartheta^{2}-z^{2}}}, \\
& w_{0}^{D}(z, \vartheta)=\frac{\Theta\left(R_{D} \sin \vartheta-|z|\right)}{\pi \sqrt{R_{D}^{2} \sin \vartheta^{2}-z^{2}}} .
\end{aligned}
$$

Comparing Eqs. (109) and (110) to the corresponding expressions for needles, Eqs. (106) and (107), reveals that the different particle geometries imprint marked differences in the functional dependence on $z$ and $\vartheta$. Clearly this can be traced back to the differences in the full weight functions for needles [Eqs. (15) and (6)] and platelets [Eqs. (19) and (20)]. Note that for the present case of vanishing particle thicknesses, $w_{2}^{D}(z, \vartheta)$ has no needle counterpart to compare with, as the needle surface vanishes.

$$
\begin{gathered}
\mathbf{w}_{v 1}^{D}(z, \vartheta)=\frac{z}{8 R_{D} \sin ^{2} \vartheta} \sqrt{\frac{R_{D}^{2} \sin ^{2} \vartheta-z^{2}}{R_{D}^{2}-z^{2}}} \mathbf{e}_{z} \\
w_{1}^{D D}\left(z, \vartheta, \vartheta^{\prime}\right)= \begin{cases}0 & \text { if } p^{2}<0 \\
\frac{1}{2} \cos \vartheta^{\prime} & \text { if } p^{2} \sin \left(\vartheta-\vartheta^{\prime}\right) \sin \left(\vartheta+\vartheta^{\prime}\right)>z^{2} \sin ^{2} \vartheta^{\prime} \\
\frac{1}{\pi p}\left(\sqrt{\left(z^{2}+p^{2}\right) \frac{\sin ^{2} \vartheta^{\prime}}{\sin ^{2} \vartheta}-p^{2}}\right. & \\
+p\left|\cos \vartheta^{\prime}\right| & \text { else } \\
\left.\times \arcsin \frac{p\left|\cot ^{\prime}\right|}{\sqrt{z^{2}+R_{D}^{2} \cos ^{2} \vartheta}}\right) & \end{cases}
\end{gathered}
$$

where $p=\sqrt{R_{D}^{2} \sin ^{2} \vartheta-z^{2}}$.

$$
w_{2}^{D D D}\left(z, \vartheta, \vartheta^{\prime}, \vartheta^{\prime \prime}\right)=\frac{8}{\pi^{2}} \sqrt{R_{D}^{2}-\frac{z^{2}}{\sin ^{2} \vartheta}} \int_{0}^{2 \pi} \mathrm{d} \varphi^{\prime \prime} u\left(\vartheta, \vartheta^{\prime}, \vartheta^{\prime \prime}, \varphi^{\prime \prime}\right) \Theta\left(R_{D}^{2} \sin ^{2} \vartheta-z^{2}\right)
$$


where

$$
u\left(\vartheta, \vartheta^{\prime}, \vartheta^{\prime \prime}, \varphi^{\prime \prime}\right)= \begin{cases}\left|\cos \vartheta^{\prime} \sin \vartheta^{\prime \prime} \sin \varphi^{\prime \prime}\right| & \text { if }\left|k\left(\vartheta, \vartheta^{\prime}, \vartheta^{\prime \prime}, \varphi^{\prime \prime}\right)\right|>1 \\ \frac{2}{\pi|\sin \vartheta|}\left(\left\{\sin ^{2} \vartheta^{\prime}\left[\sin \vartheta^{\prime \prime} \cos \vartheta \cos \varphi^{\prime \prime}-\sin \vartheta \cos \vartheta^{\prime \prime}\right]^{2}\right.\right. & \\ \left.+\sin \left(\vartheta^{\prime}+\vartheta\right) \sin \left(\vartheta^{\prime}-\vartheta\right) \sin ^{2} \vartheta^{\prime \prime} \sin ^{2} \varphi^{\prime \prime}\right\}^{1 / 2} & \text { else, } \\ \left.+\sin \vartheta \sin \vartheta^{\prime \prime} \cos \vartheta^{\prime} \sin \varphi^{\prime \prime} \arcsin k\left(\vartheta, \vartheta^{\prime}, \vartheta^{\prime \prime}, \varphi^{\prime \prime}\right)\right) & \end{cases}
$$

and

$$
k\left(\vartheta, \vartheta^{\prime}, \vartheta^{\prime \prime}, \varphi^{\prime \prime}\right)=\frac{\sin \vartheta \sin \vartheta^{\prime \prime} \cos \vartheta^{\prime} \sin \varphi^{\prime \prime}}{\sqrt{\sin ^{2} \vartheta^{\prime}\left[\left(\sin \vartheta^{\prime \prime} \cos \varphi^{\prime \prime} \cos \vartheta-\sin \vartheta \cos \vartheta^{\prime \prime}\right)^{2}+\cos ^{2} \vartheta \sin ^{2} \vartheta^{\prime \prime} \sin ^{2} \varphi^{\prime \prime}\right]}}
$$

For the remaining mixed weight functions we obtain

$$
w_{2}^{D N}\left(z, \vartheta_{D} ; \vartheta_{N}\right)=\frac{4 \cot \vartheta_{D} \cos \vartheta_{N} \sqrt{R_{D}^{2}-z^{2}}}{R_{D}^{2}-z^{2} / \sin ^{2} \vartheta_{D}} \Theta\left(R_{D} \sin \vartheta_{D}-|z|\right)
$$

\section{RESULTS}

Already the binary subsystems with platelets (leaving aside the full ternary mixture), i.e. the sphere-platelet and rod-platelet mixtures, are expected to display rich bulk and interfacial properties due to the competition between depletion and orientational order. In order to find a simple yet demanding test case for the present theory, we restrict ourselves further to the pure system of platelets. In bulk this undergoes an isotropic-nematic phase transition, where $\rho_{D}(\mathbf{r}, \boldsymbol{\Omega})=\rho_{D}=$ const in the isotropic phase, and $\rho_{D}(\mathbf{r}, \boldsymbol{\Omega})=\rho_{D}(\theta)$ in the nematic phase being peaked around the nematic director that indicates the preferred direction of alignment of the particles; $\theta$ is the angle between $\Omega$ and the nematic director. The strength of the nematic order is conveniently measured via the nematic order parameter,

$$
S=\rho_{D}^{-1} \int \frac{d^{2} \Omega}{4 \pi} \rho_{D}(\Omega) P_{2}(\cos \theta)
$$

where $P_{2}(x)=\left(3 x^{2}-1\right) / 2$ is the second Legendre polynomial.

We use the generic form of the free energy, Eq. (75), as a multiple integral over a free energy density appropriate for pure platelets, $\Phi_{D}$ as given in Eq. (78). This function depends 
on the weighted densities $n_{2}^{D}, n_{1}^{D D}$, and $n_{2}^{D D D}$, as defined in Eqs. (70) (with $\nu=2$ ), (172), and (73), respectively. The weighted densities in turn are built as convolutions of the bare platelet one-body density, $\rho(\mathbf{r}, \mathbf{\Omega})$, with the weight functions $w_{2}^{D}, w_{1}^{D D}$, and $w_{2}^{D D}$, as given in (18), (21), and (49) [and summarized in Eqs. (53), (56), and (61)], respectively. To carry out the actual calculations, we have started from the corresponding expressions in planar geometry (see Sec. VII), that we have further (numerically) reduced to $z$-independent quantities, as appropriate for the spatially homogeneous isotropic and nematic phases.

Alternatively, our FMT excess free energy functional for hard platelets can be viewed as being composed of the exact second-order virial contribution and a further (approximate) third order term that i) vanishes for configurations without common triple intersection of three platelets, and ii) is constant for configurations with common triple intersection; the constant is adjusted to give a reasonable value for the third virial coefficient. Note that the theory for the full ternary mixture has a similar structure, but features higher order (in density) contributions that arise from the finite volume, and hence finite packing fraction, of the hard spheres.

We minimize the grand potential functional

$$
\tilde{\Omega}\left(\mu_{D},\left[\rho_{D}\right]\right)=F_{\mathrm{id}}\left[\rho_{D}\right]+F_{\mathrm{exc}}\left[\rho_{D}\right]-\mu_{D} \int d \mathbf{r} \frac{d \boldsymbol{\Omega}}{4 \pi} \rho_{D}(\mathbf{r}, \boldsymbol{\Omega}),
$$

where the ideal gas contribution is given by

$$
F_{\mathrm{id}}\left[\rho_{D}\right]=\int d \mathbf{r} \frac{d \boldsymbol{\Omega}}{4 \pi} \rho_{D}(\mathbf{r}, \boldsymbol{\Omega})\left[\ln \left(\rho_{D}(\mathbf{r}, \boldsymbol{\Omega}) \Lambda_{D}^{3}\right)-1\right]
$$

where $\mu_{D}$ is the chemical potential, the dependence on temperature and volume has been suppressed in the notation, and we formally set the thermal wavelength equal to the platelet radius, $\Lambda_{D}=R_{D}$, to fix an arbitrary additive constant to the chemical potential. For a given value of the (scaled) chemical potential $\mu^{*}=\beta \mu_{D}$, we minimized $\tilde{\Omega}$ with respect to $\rho_{D}(\Omega)$ in either phase. Inserting the resulting distribution into Eq. (118) yields the grand potential of the system, and the condition for equality of the grand potential in both phases locates the phase transition.

The numerical implementation uses free minimization, i.e. no parametrized form of $\rho_{D}(\theta)$ is assumed a priori. We use an equidistant grid in $\theta$-space with 100 grid points in the interval $[0, \pi / 2]$. The integration over $\varphi^{\prime \prime}$ in Eq. (113) to obtain $w_{2}^{D D D}$ was done with 200 grid points in $[0,2 \pi]$; this only needs to be performed once at the start of the calculation. We have used 


\begin{tabular}{l|l|l|l} 
& FMT & simulation & Onsager \\
\hline$\rho_{D}^{\text {iso }} R_{D}^{3}$ & 0.418 & 0.473 & 0.667 \\
\hline$\rho_{D}^{\text {nem }} R_{D}^{3}$ & 0.460 & 0.509 & 0.845 \\
\hline$\Delta \rho_{D} R_{D}^{3}$ & 0.041 & 0.036 & 0.178 \\
\hline$S_{\text {nem }}$ & 0.492 & 0.37 & 0.781 \\
\hline
\end{tabular}

TABLE I: Results for the bulk isotropic-nematic phase transition from different approaches. Given are values for the (scaled) coexisting densities in the isotropic and nematic phase, $\rho_{D}^{\text {iso }} R_{D}^{3}$ and $\rho_{D}^{\text {nem }} R_{D}^{3}$, respectively, the (scaled) density jump, $\Delta \rho_{D} R_{D}^{3}=\left(\rho_{D}^{\text {nem }}-\rho_{D}^{\text {iso }}\right) R_{D}^{3}$, and the value of the nematic order parameter in the coexisting nematic phase, $S_{\text {nem }}$, as obtained from FMT, and the simulation results by Frenkel and Eppenga [48], as well as theoretical results obtained from the Onsager second virial functional for hard platelets.

alternatively fixed-point iteration or simulated annealing to minimize the density profile; results from both approaches were consistent. Up to 1000 steps were sufficient to obtain convergence.

The resulting values of the densities in the coexisting isotropic and nematic phases, $\rho_{D}^{\text {iso }}$ and $\rho_{D}^{\text {nem }}$, respectively, as well as the value of the order parameter in the coexisting nematic phase, $S_{\text {nem }}$, are in good agreement with Frenkel and Eppenga's classic simulation results 48], see Tab. [. The FMT values somewhat underestimate the coexistence densities and

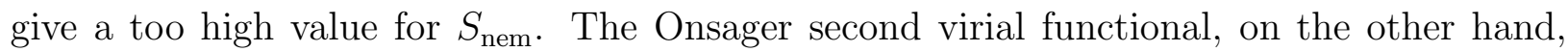
overestimates significantly the coexistence densities as well as $S_{\text {nem }}$. Hence the actual phase transition, as characterized by the jump in coexistence densities and in order parameter, is weaker than predicted by the Onsager treatment. To further illustrate our findings, we plot in Fig. [7 the variation of $S$ with $\mu^{*}$ and in Fig. 8 the variation of $S$ with $\rho_{D} R_{D}^{3}$. We also plot the behavior of the metastable nematic branch, i.e. for statepoints where the nematic phase remains locally stable against small fluctuations, although globally the grand potential for the isotropic phase is the lower one. It is apparent that the value of $S_{\text {nem }}$ very sensitively depends on the precise location of the phase transition. Taking this into account we find the overall the agreement of the FMT results with simulation data to be very reasonable, and to give confidence both into applications to interfacial phenomena of platelets, as well as into the accuracy of the FMT for the full ternary mixture. 


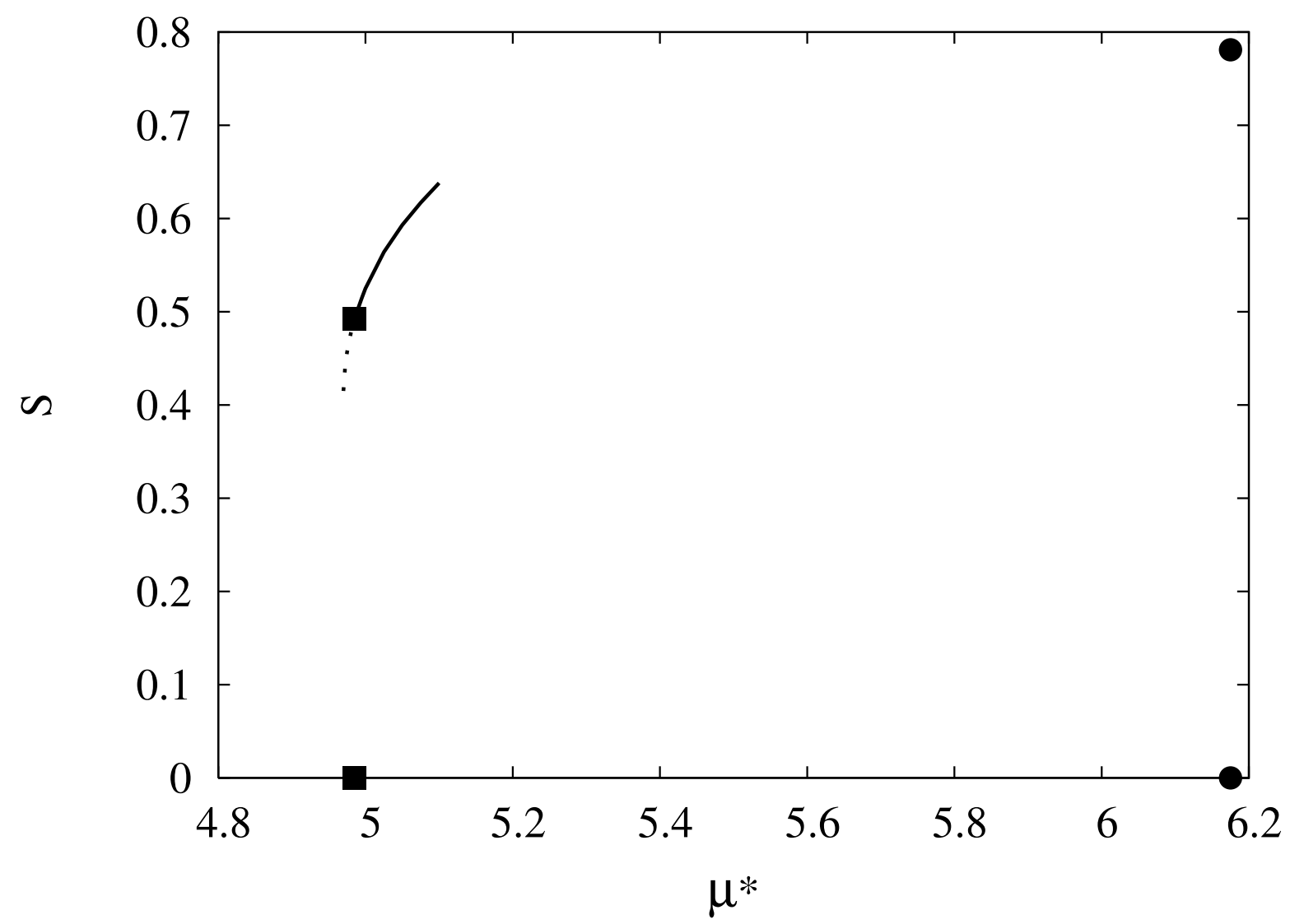

FIG. 7: Nematic order parameter $S$ for a bulk system of pure hard platelets as a function of the (scaled) chemical potential, $\mu^{*}$. Shown are results from FMT in the stable (solid line) and metastable (dashed line) nematic. Values in the coexisting nematic and isotropic phases, $S_{\text {nem }}$ and $S_{\text {iso }}=0$, are shown as obtained from FMT (squares) and the Onsager-type second virial treatment (dots).

\section{CONCLUSIONS}

In conclusion we have derived a geometry-based density functional theory for hard body mixtures of spheres, platelets and needles. Both the needles and the (circular) platelets possess vanishing thickness and hence constitute the simplest examples of prolate and oblate model particles, respectively. Our treatment of the mixture is based on the so-called deconvolution of the Mayer bond into single-particle functions which vanish beyond the extent of the particle. The Mayer bonds are recovered upon convolution of the single-particle functions. The construction of the full functional relies further on controlling the third virial level and on Rosenfeld's scaled-particle treatment. In order to facilitate future applications, 


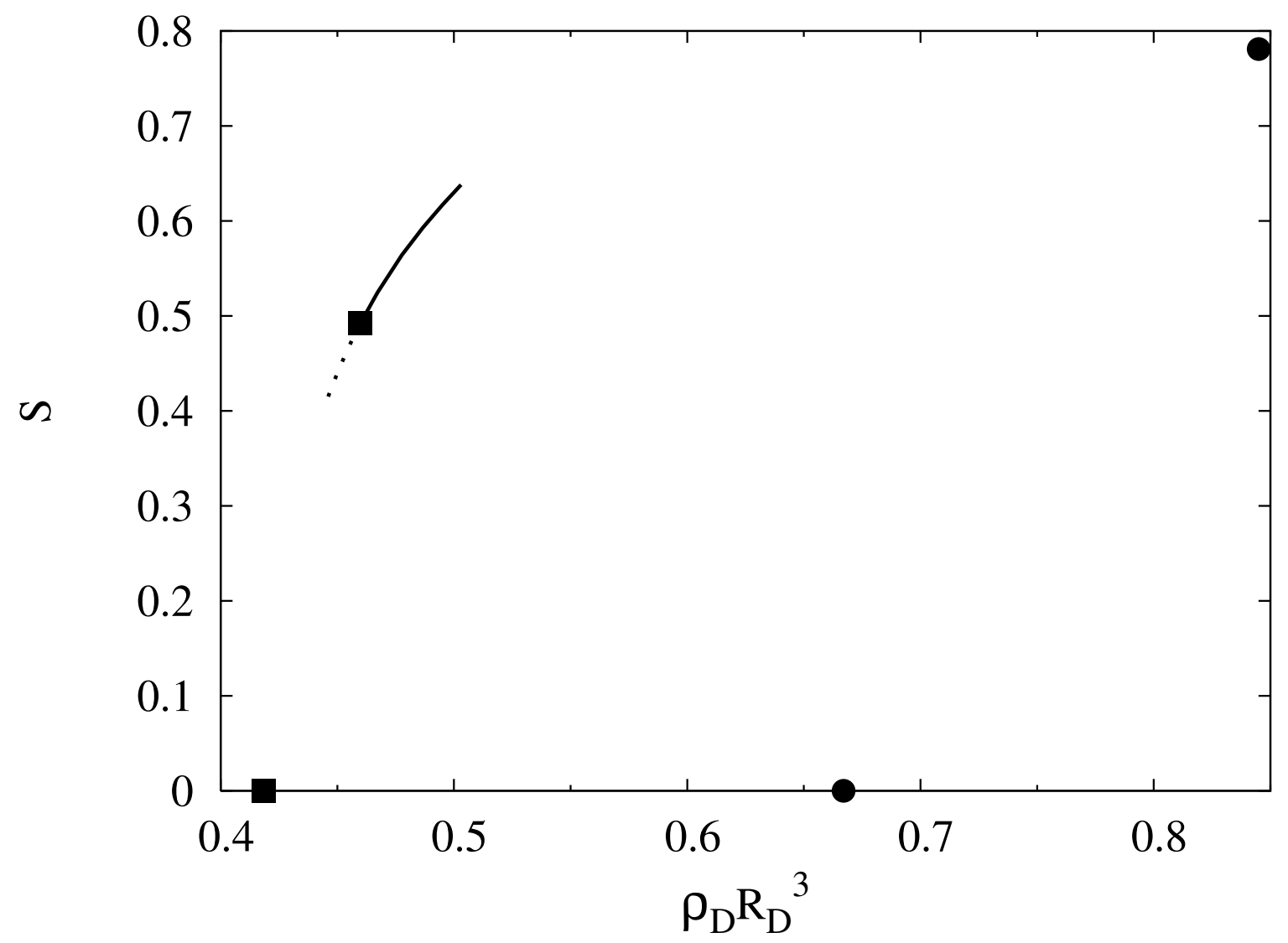

FIG. 8: Same as Fig. [7 but as a function of the (scaled) density of platelets $\rho_{D} R_{D}^{3}$. Note the considerable reduction of the coexistence density gap obtained from FMT as compared to the Onsager-like treatment.

like wetting of planar walls or capillary phenomena in planar slits, we have given explicit reduced expressions for the relevant quantities in planar geometry.

In a recent contribution Harnau and Dietrich propose and apply a DFT for binary plateletsphere mixtures [49]. They obtain a platelet-sphere functional by starting from the rodsphere functional of Refs. 38, 39], and in particular from the explicit expressions for the needle weight functions in planar and uniaxial geometry Modifying the definition of the relevant angle between the particle orientation and the $z$-axis the rod weight functions are taken to play the role of platelet weight functions. The resulting excess free energy functional is linear in the platelet density, limiting the theory to small densities of platelets. Recall that for binary mixtures where one component (the depletant) is ideal, the absence of higher than linear order terms in the density distribution of this component is a good 
approximation. Examples are the above rod-sphere mixture and the Asakura-Oosawa model of colloid-polymer mixtures, where the polymers are described as non-interacting spheres. While in these cases the pure depletant system is an ideal gas, pure platelets constitute an interacting system. Hence higher than linear order terms in the platelet density should only be irrelevant at low platelet densities. The current theory is in accordance with scaledparticle theory [54] and yields second and third-order contributions to the excess free energy, but higher order terms are absent. This behavior can be traced back to the vanishing volume of the platelets.

In the present work we have shown explicitly how our DFT for the sphere-platelet mixture reduces to the correct low-density limit. In contrast to the procedure in Ref. [49], we have treated the full three-dimensional problem and have obtained, up to a defect already present for two-dimensional hard disks, the deconvolution of the sphere-platelet Mayer bond and hence the appropriate platelet weight functions. Projecting those to planar and uniaxial symmetry (appropriate for fluid states at a planar smooth wall like investigated in Ref. 49]) reveals that the expresssions differ markedly from those for sphere-rod mixtures. This might come as no surprise given the fact that the genuine shapes of the particles are one of the building blocks of the geometry-based DFT. An immediate consequence is that the platelet weight functions, and hence the form of the excess free energy functional, also differ from the expressions used in Ref. [49].

Our successful description of the bulk isotropic-nematic transition of hard platelets can be viewed as the first genuine FMT treatment of liquid crystalline ordering in a continuum model (without any interpolation as e.g. in Ref. [23]). Possible future applications of our theory include capillary and wetting phenomena, influence of gravity or other external fields, and the study of free interfaces between demixed (and possibly liquid crystalline) phases. Further testing the accuracy of the theory, i.e. for the predictions of the bulk di-

rect correlation functions, see e.g. [55], against results from computer simulations is clearly desirable.

\section{Acknowledgments}

Professor Siegfried Dietrich and Dr. Ludger Harnau are acknowledged for useful discussions. We thank Dr. Marjolein Dijkstra and Dr. René van Roij for stimulating exchange and 
for communicating their unpublished results to us. Dr. Andrew Archer is acknowledged for valuable comments on the manuscript. This work is supported by the SFB-TR6 "Colloidal dispersions in external fields" of the German Science Foundation (Deutsche Forschungsgemeinschaft).

[1] M. Dijkstra, J. P. Hansen, and P. A. Madden, Phys. Rev. E 55, 3044 (1997).

[2] M. Dijkstra, J. P. Hansen, and P. A. Madden, Phys. Rev. Lett. 51, 5891 (1995).

[3] D. van der Beek, A. V. Petukhov, S. M. Oversteegen, G. J. Vroege, and H. N. W. Lekkerkerker, Eur. Phys. J. E 16, 253 (2005).

[4] S. M. Oversteegen, J. G. E. J. Wijnhoven, and H. N. W. Lekkerkerker, J. Phys. Chem. B 108, 18158 (2004).

[5] T. G. Mason, Phys. Rev. E 66, 060402(R) (2002).

[6] E. S. H. Leach and J. S. van Duijneveldt, Langmuir 21, 3821 (2005).

[7] L. Harnau and S. Dietrich, Phys. Rev. E 65, 021505 (2002).

[8] L. Harnau, D. Costa, and J. P. Hansen, Europhys. Lett. 53, 729 (2001).

[9] L. Harnau and S. Dietrich, Phys. Rev. E 66, 051702 (2002).

[10] L. Harnau, D. Rowan, and J. P. Hansen, J. Chem. Phys. 117, 11359 (2002).

[11] M. Bier, L. Harnau, and S. Dietrich, Phys. Rev. E 69, 021506 (2004).

[12] L. Harnau, F. Penna, and S. Dietrich, Phys. Rev. E 70, 021505 (2004).

[13] S. M. Oversteegen and H. N. W. Lekkerkerker, J. Chem. Phys. 120, 2470 (2004).

[14] S. M. Oversteegen and H. N. W. Lekkerkerker, Physica A 341, 23 (2004).

[15] L. Harnau and S. Dietrich, Phys. Rev. E 69, 051501 (2004).

[16] D. van der Beek, T. Schilling, and H. N. W. Lekkerkerker, J. Chem. Phys. 121, 5423 (2004).

[17] H. H. Wensink and H. N. W. Lekkerkerker, Europhys. Lett. 66, 125 (2004).

[18] R. Evans, Adv. Phys. 28, 143 (1979).

[19] R. Evans, in Fundamentals of Inhomogeneous Fluids, edited by D. Henderson (Dekker, New York, 1992), Chap. 3, p. 85.

[20] Y. Rosenfeld, Phys. Rev. Lett. 63, 980 (1989).

[21] Y. Rosenfeld, Phys. Rev. E 50, R3318 (1994).

[22] Y. Rosenfeld, Mol. Phys. 86, 637 (1995). 
[23] G. Cinacchi and F. Schmid, J. Phys.: Condensed Matter 14, 12223 (2002).

[24] J. A. Cuesta, Phys. Rev. Lett. 76, 3742 (1996).

[25] J. A. Cuesta and Y. Martinez-Raton, Phys. Rev. Lett. 78, 3681 (1997).

[26] J. A. Cuesta and Y. Martinez-Raton, J. Chem. Phys. 107, 6379 (1997).

[27] Y. Martinez-Raton and J. A. Cuesta, Phys. Rev. Lett. 89, 185701 (2002).

[28] Y. Martinez-Raton and J. A. Cuesta, J. Chem. Phys. 118, 10164 (2003).

[29] A. Chamoux and A. Perera, J. Chem. Phys. 104, 1493 (1996).

[30] A. Chamoux and A. Perera, Phys. Rev. E 58, 1933 (1998).

[31] A. Perera, J. Mol. Liq. 109, 73 (2004).

[32] P. Bryk, Phys. Rev. E 68, 062501 (2004).

[33] P. Bryk and R. Roth, Phys. Rev. E 71, 011510 (2005).

[34] M. S. Wertheim, Mol. Phys. 83, 519 (1994).

[35] M. S. Wertheim, Mol. Phys. 89, 989 (1996).

[36] M. S. Wertheim, Mol. Phys. 89, 1005 (1996).

[37] P. Bolhuis and D. Frenkel, J. Chem. Phys. 101, 9869 (1994).

[38] M. Schmidt, Phys. Rev. E 63, 050201(R) (2001).

[39] J. M. Brader, A. Esztermann, and M. Schmidt, Phys. Rev. E 66, 031401 (2002).

[40] A. Esztermann and M. Schmidt, Phys. Rev. E 70, 022501 (2004).

[41] P. G. Bolhuis, J. M. Brader, and M. Schmidt, J. Phys.: Condensed Matter 48, S3421 (2003).

[42] M. Schmidt and A. R. Denton, Phys. Rev. E 65, 021508 (2002).

[43] M. Schmidt and J. M. Brader, J. Chem. Phys. 119, 3495 (2003).

[44] M. Schmidt and M. Dijkstra, J. Chem. Phys. 121, 12067 (2004).

[45] L. L. Rasmussen and D. W. Oxtoby, J. Phys.: Condensed Matter 14, 12021 (2002).

[46] J. A. Cuesta, Y. Martinez-Raton, and P. Tarazona, J. Phys.: Condensed Matter 14, 11965 (2002).

[47] P. Tarazona and Y. Rosenfeld, Phys. Rev. E 55, R4873 (1997).

[48] D. Frenkel and R. Eppenga, Phys. Rev. Lett. 49, 1089 (1982).

[49] L. Harnau and S. Dietrich, Phys. Rev. E 71, 011504 (2005).

[50] S. M. Oversteegen and R. Roth, J. Chem. Phys. 122, 214502 (2005).

[51] Y. Rosenfeld, M. Schmidt, H. Löwen, and P. Tarazona, Phys. Rev. E 55, 4245 (1997).

[52] P. Tarazona, Phys. Rev. Lett. 84, 694 (2000). 
[53] R. Roth, R. Evans, A. Lang, and G. Kahl, J. Phys.: Condensed Matter 14, 12063 (2002).

[54] J. A. Barker and D. Henderson, Rev. Mod. Phys. 48, 587 (1976).

[55] N. H. Phuong, G. Germano, and F. Schmid, Comp. Phys. Comm. 147, 350 (2002).

[56] We use $D$ and $D^{\prime}$ to refer both to the platelets and the points of their origins. Consider the triangle $\Delta$ between the center of platelet $D$, the intersection $I$ of platelet $D^{\prime}$ with the rim of $D$, and the center of $D^{\prime}$ projected onto the $x-y$ plane (call this point $A$ ). This triangle has by construction two known sides, $R_{D}$ and $r$. The length of the third side of $\Delta$ can be calculated by the cosine theorem from the angle $\angle A D I$. Using the Pythagorean theorem on the triangle between $I$ and the real and projected centers of $D^{\prime}$, the length $\left|\overline{D^{\prime} I}\right|$ can be calculated and compared to the radius of $D^{\prime}, R_{D}$. This is accomplished by the first step function. In order to calculate the angle needed for the cosine theorem, consider $\Delta$ and the right-angled triangle $\Delta^{\prime}$ that is obtained by continuing $\overline{A I}$ over $I$ to form a right angle at the new point $B$. Then, $|\overline{A B}|=|r \cos \bar{\varphi}|$ and $|\overline{A I}|=|z \bar{z}| / \sqrt{1-\bar{z}^{2}}$. Therefore, the arccos equals the angle $\angle D I B$, and we obtain the angle $\angle A D I$ by adding $\bar{\varphi}$. The second step function in Eq. (26) checks if the plane defined by $D^{\prime}$ intersects with the rim of $D$. 